\title{
Reversible Epigenetic Alterations Regulate Class I HLA Loss in Prostate Cancer
}

\section{Tamara Rodems}

University of Wisconsin

\section{Erika Henninger}

University of Wisconsin, Madison

Charlotte Stahlfeld

University of Wisconsin, Madison

Cole Gilsdorf

University of Wisconsin, Madison

Kristin Carlson

University of Wisconsin, Madison

Madison Kircher

University of Wisconsin, Madison

Anupama Singh

University of Wisconsin, Madison

Timothy Krueger

University of Wisconsin, Madison

David Beebe

University of Wisconsin-Madison https://orcid.org/0000-0002-0415-9006

David Jarrard

University of Wisconsin School of Medicine and Public Health

Douglas McNeel

University of Wisconsin Comprehensive Cancer Center https://orcid.org/0000-0003-1471-6723

Michael Haffner

Fred Hutchinson Cancer Research Center

Joshua Lang ( $\square$ jmlang@medicine.wisc.edu )

University of Wisconsin

\section{Article}

Keywords: expression, cells, prostate, cancer, methylation, loss, gene

Posted Date: November 11th, 2021 
DOl: https://doi.org/10.21203/rs.3.rs-894353/v1

License: (c) (1) This work is licensed under a Creative Commons Attribution 4.0 International License. Read Full License

Version of Record: A version of this preprint was published at Communications Biology on September 1st, 2022. See the published version at https://doi.org/10.1038/s42003-022-03843-6. 


\section{Reversible Epigenetic Alterations Regulate Class I HLA Loss in Prostate Cancer}

Authors: Tamara S. Rodems ${ }^{1, \#}$, Erika Heninger ${ }^{1,2, \#}$, Charlotte N. Stahlfeld ${ }^{1}$, Cole S. Gilsdorf ${ }^{1}$, Kristin Carlson ${ }^{1}$, Madison R. Kircher ${ }^{1}$, Anupama Singh ${ }^{1,2}$, Timothy E. G. Krueger $^{1}$, David J. Beebe ${ }^{1,3}$, David F. Jarrard ${ }^{1,4}$, Douglas G. McNeel ${ }^{1}$, Michael C. Haffner ${ }^{5,6,7}$, and Joshua M. Lang ${ }^{1,2}$

\section{Affiliations:}

${ }^{1}$ University of Wisconsin Carbone Cancer Center, University of Wisconsin, Madison, 1111 Highland Ave., Madison, WI 53705

${ }^{2}$ Department of Medicine, University of Wisconsin, Madison, 1111 Highland Ave., Madison, WI 53705

${ }^{3}$ Department of Biomedical Engineering, University of Wisconsin, Madison, 1111 Highland Ave., Madison, WI 53705

${ }^{4}$ Department of Surgery, University of Wisconsin, Madison, 1111 Highland Ave., Madison, WI 53705

${ }^{5}$ Divisions of Human Biology and Clinical Research, Fred Hutchinson Cancer Research Center, 1100 Fairview Ave, N., Seattle, WA 98109

${ }^{6}$ Department of Pathology, University of Washington, 1959 NE Pacific St., Seattle, WA 98195

${ }^{7}$ Department of Pathology, Johns Hopkins School of Medicine, 600 N Wolfe St., Baltimore, MD 21287 \# These authors contributed equally

Corresponding Author: Joshua M. Lang, Department of Medicine, Carbone Cancer Center, University of Wisconsin Madison 1111 Highland Avenue, WIMR 7151, Madison, WI 53705; Phone: 608-262-0705; Fax: 608-265-0614; Email:

jmlang@medicine.wisc.edu

Conflict of Interest: Joshua M. Lang and David J. Beebe hold equity in Salus Discovery, which has licensed some of the technology described in the manuscript. David J. Beebe also holds equity in BellBrook Labs LLC, Tasso Inc., Stacks to the Future LLC, Lynx Biosciences LLC, Onexio Biosystems LLC, Turba LLC, and is a consultant for Abbott Laboratories. 


\section{ABSTRACT}

Downregulation of HLA class I (HLA-I) impairs immune recognition and surveillance in prostate cancer and may underlie the ineffectiveness of checkpoint blockade. However, the molecular mechanisms regulating HLA-I loss in prostate cancer have not been fully explored. Here, we conducted a comprehensive analysis of HLA-I genomic, epigenomic and gene expression alterations in primary and metastatic human prostate cancer. Loss of HLA-I gene expression was associated with repressive chromatin states including DNA methylation, histone $\mathrm{H} 3$ tri-methylation at lysine 27 , and reduced chromatin accessibility. Pharmacological DNMT and HDAC inhibition decreased DNA methylation and increased $\mathrm{H} 3$ lysine 27 acetylation and resulted in re-expression of HLA-I on the surface of tumor cells. Re-expression of HLA-I on LNCaP cells by DNMT and HDAC inhibition increased activation of co-cultured PSMA $27-38$-specific CD8+ T-cells.

Methylated HLA-I was detected in HLA-I low circulating tumor cells (CTCs), which may serve as a biomarker for identifying patients who would benefit from epigenetic targeted therapies.

\section{INTRODUCTION}

Approximately 30,000 men die of metastatic prostate cancer per year in the United States and the incidence of men presenting with metastatic disease is rising ${ }^{1,2}$. There is a critical need to identify the molecular drivers that contribute to prostate cancer growth and metastasis. Immune evasion is one of the hallmarks of cancer pathogenesis and can be therapeutically targeted by immunotherapies that augment T-cell recognition and lysis of tumor cells. ${ }^{3-5}$. However, display of a functional major histocompatibility complex class I (MHC-I) is required for recognition of tumor cells by cytotoxic $T$ lymphocytes (CTLs). Lack of MHC-I display at the cell surface reduces tumor immunogenicity and drives resistance to immune checkpoint inhibitors ${ }^{6-9}$. MHC-I is a multimeric protein composed of a class I human leukocyte antigen (HLA-I) protein (A, B, or C), beta-2-microglobulin (B2M), and a peptide derived from an intracellular protein ${ }^{10}$. Downregulation of the MHC-I components has been proposed as a mechanism of immune evasion in numerous cancer types, including prostate cancer 6,11,12. Downregulation of HLA-I was observed in $~ 70 \%$ of primary prostate tumors with complete loss in up to $34 \%$ of primary tumors and $80 \%$ of metastatic lesions ${ }^{13,14}$. The molecular alterations that lead to HLA-I downregulation in prostate cancer remain largely unknown.

Recent findings have pointed to epigenetic mechanisms as drivers of prostate

cancer progression ${ }^{15,16}$. Overexpression of epigenetic modifying proteins, including the de novo methyltransferases, DNMT3A and DNMT3B, and class I histone deacetylases, 
HDAC1, HDAC2, and HDAC3, has been implicated in altering epigenetic programs and contributing to metastasis in prostate cancer ${ }^{17,18}$. Studies in esophageal squamous cell carcinoma and gastric cancer found that DNA methylation contributed to HLA-I downregulation, however epigenetic regulation of HLA-I has not yet been explored in prostate cancer ${ }^{19,20}$. Inhibition of DNMT and HDAC proteins has been proposed as a therapeutic strategy in prostate cancer, though minimal clinical success has been observed in solid tumors ${ }^{21}$. Despite this, there is promising evidence for the usefulness of epigenetic therapies in combination with immunotherapy ${ }^{22}$. There remains a need to better understand the interplay between epigenetic and immune functions in cancer cells and for accessible biomarkers for both predicting treatment benefit and monitoring treatment response ${ }^{22}$. We have previously demonstrated that modulating the activity of DNMT and HDAC proteins in prostate cancer cell lines and ex vivo human prostate tissue induced the expression of cancer testis antigens (CTAs), which have been proposed as targets for tumor vaccine therapies ${ }^{23}$. Taking advantage of the ability of epigenetic mechanisms to modulate aspects of the immune response, such as HLA-I expression, may improve the efficacy of certain immunotherapies.

In this report, we demonstrate that transcriptional downregulation of HLA-I is coordinated by epigenetic silencing mechanisms, which can be reversed to functionally re-express HLA-I and restore MHC-I dependent T-cell activation. Additionally, we show that methylation in the HLA-I genes can be detected in prostate cancer circulating tumor cells (CTCs) with low MHC-I protein expression, which is a potential predictive biomarker to identify patients who may benefit from epigenetic therapies and monitor treatment response.

\section{RESULTS}

\section{HLA-I Gene Expression is Downregulated in a Subset of Prostate Cancers}

Previous studies have shown widespread HLA-I downregulation at the protein level in prostate cancer tissues when compared to normal tissue ${ }^{12-14,24}$. To confirm that this phenomenon occurs at the transcriptional level, we analyzed changes in HLA-I gene expression in prostate cancer compared to normal tissue in two data sets that included normal tissue in their gene expression analysis: the TCGA PanCancer Prostate Adenocarcinoma (PRAD) data set, which contains only primary tumor samples, and a data set from Taylor et al, which contained both primary and metastatic tumor samples 25. Gene expression in the PRAD data set was generated with RNA-seq and gene expression in the Taylor data set was generated with microarrays. Data was converted into z-scores comparing tumor samples to the normal samples from the respective study in order to make comparisons between the two different experimental systems, 
however appropriate precaution should be used for any primary:primary comparisons due to the different methods of data acquisition.

We examined both the mean z-score in the population as well as the number and percentage of samples that were significantly up- and downregulated based on a confidence level of $95 \%$ (Figure $1 \mathrm{~A}$, Table S1). In the two primary data sets, only HLA-A showed noticeable negative shift in mean z-score, indicating a population level downregulation of gene expression compared to normal samples. However, for all three genes, there was a subset of the population ranging from $5 \%$ to $14 \%$ with significantly reduced gene expression in the primary tumors (Table S1). HLA-A, HLA-B, and HLA-C were downregulated in $58 \%-63 \%$ of the metastatic samples and had significantly negative shifts in mean z-score at the population level. Additionally, expression of each HLA-I gene was highly positively correlated with the other HLA-I genes, suggesting no compensation from the other genes takes place if one is lost (Figure 1B).

Significant downregulation of HLA-I was also associated with decreased time to biochemical recurrence (two occurrences of PSA $\geq 0.2 \mathrm{ng} / \mathrm{mL}$ ) after radical prostatectomy when compared to all other patients or patients with significantly upregulated HLA-I expression (Figure 1C,D). These data show that HLA-I may be transcriptionally downregulated in a subset of primary prostate cancers and the majority of metastatic prostate cancers and loss of HLA-I gene expression may be a risk factor for biochemical recurrence.

\section{Aberrantly Expressed DNMT and Class I HDAC Genes are Correlated to HLA-I in Prostate Cancer}

Analysis of copy number alterations and mutations in the HLA-I genes revealed that the majority of HLA-I gene expression changes in prostate cancer are unlikely to be driven by genomic alterations due to low alteration frequency and lack of correlation to gene expression changes (Figure S1). Previous studies have identified epigenetic mechanisms, including DNA methylation and histone modifications, as driving forces in prostate cancer biology. DNMTs and class I HDACs have been widely studied as potential therapeutic targets as well as mechanistic drivers for regulation of gene expression. We hypothesized that the DNMTs and class I HDACs could promote immune evasion by downregulating HLA-I. We analyzed the expression of DNMT1, DNMT3A, DNMT3B, and the class I HDACs HDAC1, HDAC2, HDAC3, and HDAC8 in the PRAD and Taylor data sets and their correlation to HLA-I expression in prostate cancer. Table S1 summarizes the number and percentage of patients in each study who showed up- and downregulation of each of these genes. The de novo DNMTs and class I HDAC genes tended to be upregulated more often than downregulated with the exception of HDAC8, which was upregulated in $25 \%$ the samples in the PRAD data set, but only in 1 sample from the Taylor data set. An analysis of the mean z-score in each data set revealed that $D N M T 3 A, D N M T 3 B$ and $H D A C 1 / 2 / 3$ tended to have positively 
shifted z-scores indicating an increase in mean gene expression in the tumor populations (Figure S2A).

To investigate how the expression of these various genes associate with HLA-I expression, we calculated Pearson $r$ values and $p$-values for the correlation between HLA-I and DNMT and HDAC genes in each data set (Figure 1B, Table S2). Correlation patterns varied among the data sets, however we found that $D N M T 3 B$ was highly negatively correlated to HLA-I gene expression in all three data sets and HDAC2 was the most negatively correlated to HLA-I gene expression in the metastatic samples (Figure 1E, S1B). DNMT3A was also negatively correlated to HLA-I gene expression in the PRAD data set. HDAC3 and HDAC8 gene expression was positively correlated to HLA-I gene expression in the Taylor data sets, though showed weak to no correlation in the PRAD data set. Overall, the correlation of DNMT3B and HDAC2 to HLA-I gene expression along with overexpression of these gene families, suggests a key role for epigenetic modification of DNA and histones in HLA-I downregulation.

\section{HLA-I CpG Islands are Methylated in Prostate Cancer}

We next sought to investigate the DNA methylation signatures of the HLA-I genes in primary and metastatic prostate cancer biopsies. We analyzed the level of methylation at individual probes from the Illumina 450K methylation array within the HLA-I CpG islands in normal prostate tissue vs. prostate adenocarcinoma samples in the PRAD data set (Figure $S 3 A, B)$. Probe locations are shown in Figure 2A. Every probe located within the HLA-A CpG island showed higher methylation levels in prostate tumor samples compared to normal. There were also multiple probes located within $H L A-B$ and $H L A-C$ with significantly higher levels of methylation compared to normal samples. Notably, the probe located within $50 \mathrm{bp}$ upstream of the transcription start site for both $H L A-A$ and $H L A-C$ had the most significant increase in methylation in prostate tumors compared to normal samples (Figure S3C). In order to examine overall methylation levels in the promoter and intragenic regions of the $\mathrm{CpG}$ islands, average methylation level at the probes within these two regions was analyzed for tumor and normal samples (Figure 2B). The average level of methylation was significantly higher in the tumor samples for all regions except the HLA-B promoter. $H L A-A$ had the highest overall methylation level and all three genes tended to have higher methylation in the intragenic regions compared to the promoters.

We then explored whether the patients with increased levels of methylation in HLA-I had corresponding decreases in HLA-I gene expression. Correlations between matched patient gene expression from RNA-seq data and the methylation score at each probe were calculated (Table S3). Significant negative correlations were found in 10/10 HLA-A probes, 5/13 HLA-B probes, and 10/11 HLA-C probes in the tumor samples, and only $1 / 10,3 / 13$ and $2 / 11$ for $H L A-A, H L A-B$ and $H L A-C$ normal samples respectively. While many of these correlations have reached statistical significance, the Pearson $r$ 
values are relatively weak, which may be due the smaller subset of patients in these cohorts that have significantly reduced HLA-I gene expression, as summarized in Table S1. To further investigate the association between gene expression and methylation in HLA-I genes, we separated the samples into three groups: high, medium, and low expression. High and low expression were defined by having a significant z-score using a $95 \%$ confidence level relative to normal samples, with all non-significant samples placed into the medium category. We then compared methylation levels in the stratified samples to normal samples at each probe and the average methylation across the promoter and intragenic regions (Figure 2C, S3D, S3E). Methylation in HLA-A and HLA$C$ promoter and intragenic regions was significantly higher in the samples with low HLAI expression compared to samples with high HLA-I expression. Samples expressing medium levels of $\mathrm{HLA}-\mathrm{I}$ also tended to have lower methylation levels compared to samples expressing low levels of HLA-I. The average methylation levels in the HLA-B promoter and intragenic regions did not show strong significant differences between gene expression groups. However, when we looked at individual $H L A-B$ probes, we saw similar trends of low gene expression being associated with higher methylation levels (Figure S2F). This analysis demonstrates a role for DNA methylation in regulating HLA-I expression in primary prostate cancer.

We next evaluated the presence of HLA-A methylation in primary and metastatic tumor samples (Figure 2D). We employed COMPARE-MS, a method that has been previously used to measure methylation in prostate cancer biopsies ${ }^{26-30}$. HLA-A methylation was either not detected or detected at very low levels in benign samples. We were able to detect HLA-A methylation in a small subset of primary tumors and in the majority of metastatic sites. The level of detection tended to be similar across different metastatic sites from the same patient. The increase in frequency of detection of $H L A-A$ in metastatic samples suggests $H L A-A$ methylation maybe selected for during metastasis. Taken together with the low HLA-A gene expression in metastatic samples shown in Figure 1A, these analyses suggest a significant role for DNA methylation in $H L A-A$ transcriptional downregulation in patients with metastatic prostate cancer.

\section{Decreased Chromatin Accessibility is Associated with HLA-I Downregulation in Prostate Cancer}

Studies on histone modifications in prostate cancer have been conducted largely at the global level with studies typically measuring overall levels of histone modification abundance ${ }^{31,32}$. HLA-I specific histone modification signatures have not been explored in patients with prostate cancer. However, we can make inferences about epigenetic regulation of HLA-I in prostate cancer using ATAC-seq data from the TCGA PanCancer study. We analyzed the ATAC-seq signals in available primary prostate samples from the PRAD data set at two genomic locations: a proximal enhancer region and the promoter. We then compared the changes in chromatin accessibility at these regions to 
matched HLA-I gene expression. While the sample size was small, those with high HLA-I gene expression tended to have higher scores for chromatin accessibility (Figure 2E). HLA-A gene expression was significantly positively correlated with ATAC-seq signal at the proximal enhancer region and HLA-C gene expression was significantly positively correlated with ATAC-seq signal at the promoter (Figure 2F). All other ATACseq signals were also positively correlated with HLA-I gene expression, but did not reach significance, likely due to the small sample size. This preliminary analysis suggests that HLA-I downregulation is associated with a decrease in accessible chromatin in key regulatory regions.

\section{HLA-I Downregulation is Associated with Increased DNA Methylation and Repressive Histone Modifications in Prostate Cancer Cell Lines}

We next evaluated epigenetic signatures in prostate cancer cell lines where HLA-I expression is downregulated. HLA-I protein and gene expression were downregulated in the prostate cancer cell lines LNCaP, 22rv1, PC3, and LAPC4 when compared to a normal prostate epithelial cell line, RWPE1 (Figure 3A-C, S4). Methylation of HLA-I was evaluated using MBD2-based enrichment of methylated DNA followed by qPCR (Figure 3D). Primers were designed to target regions of the HLA-I genes that were identified as being differentially methylated in patient samples, including the distal and proximal promoter as well as two intragenic regions (IG) near Exon1/Intron1 (IG 1) and Exon2/Intron2 (IG 2) (Figure 3E). LAPC4 had the highest level of methylation in all three HLA-I genes. PC3 and LNCaP also had increased methylation in certain gene regions compared to RWPE1. 22rv1 had the lowest overall methylation of the cancer cell lines. Methylation in IG2 tended to be the most enriched in cancer cell lines and overall, methylation in the cancer cell lines was increased in at least 2 of the evaluated gene regions for each gene (Figure 3F). Overall, the methylation landscape in these four prostate cancer cell lines is comparable to the signatures found in patient samples.

Since HLA-I gene expression was associated with a less accessible chromatin state (Figure 2D), we hypothesized that repressive histone signatures may also be present in the HLA-I genes in prostate cancer cell lines. Chromatin immunoprecipitation (ChIP) was performed using antibodies targeting acetylated (H3K27ac), a marker of active transcription, and tri-methylated (H3K27me3) lysine 27 on histone $\mathrm{H} 3$, a marker of transcriptional repression. Primers were designed to locations near a peak in H3K27ac signature in GM12878, a lymphoblastoid cell line, determined by ChIP-seq from the ENCODE consortium ${ }^{33}$ (Figure 3E). Overall, the prostate cancer cell lines showed significant decreases in $\mathrm{H} 3 \mathrm{~K} 27 \mathrm{ac}$ and increases in $\mathrm{H} 3 \mathrm{~K} 27 \mathrm{me} 3$ when compared to RWPE1 (Figure 3G,H). The cell lines 22rv1 and LAPC4 had particularly low levels of lysine 27 acetylation in HLA-I. LAPC4 and 22rv1 also had the highest overall level of trimethylation. This strong repressive signature in 22rv1 may explain why HLA-I expression is so low in these cells, even though DNA methylation was not very high, 
suggesting that individual tumors may regulate HLA-I by different epigenetic mechanisms.

We generated correlation matrices for HLA-I protein expression, gene expression, methylation, and histone modifications to examine the relationships between each of these measures in the prostate cell lines (Figure 3I). We found that protein and gene expression were highly positively correlated in each gene. Methylation within each region tended to be positively correlated with methylation in other regions and with $\mathrm{H} 3 \mathrm{~K} 27$ tri-methylation, and negatively correlated with H3K27 acetylation, indicating co-occurrence of these epigenetic signatures. The pattern of correlation between methylation signatures in each gene region and HLA-I expression was heterogeneous. We found that methylation within the IG 1 and IG 2 region of $H L A-A$ and $H L A-C$ was negatively correlated with corresponding gene and protein expression. $H L A-$ $B$ IG 2 methylation was also negatively correlated with HLA-B gene and protein expression. The strongest correlations were found in the histone modification group. H3K27 acetylation was strongly positively correlated with gene and protein expression and H3K27 tri-methylation was strongly negatively correlated with gene and protein expression for all three HLA-I genes. This analysis demonstrates that strong repressive epigenetic signatures are enriched at the HLA-I genes and correlated to HLA-I gene expression in prostate cancer cell lines.

\section{DNMT and HDAC Inhibition Induces HLA-I Expression and Reverses Repressive Epigenetic Signatures in vitro}

To further investigate epigenetic regulation of HLA-I, we pharmacologically inhibited DNMT and HDAC activity in prostate cancer cell lines and measured HLA-I protein and gene expression in response. We treated LNCaP, 22rv1, PC3, and LAPC4 cells with DNMT inhibitors SGI-110 (SGI) and 5-aza-2-deoxycytidine (5AZA2) alone and in combination with the HDAC inhibitor LBH-589 (LBH). We found that the cancer cell lines all responded to at least one treatment condition (Figure 4A-D). The combination treatment was the most effective to restore HLA-I expression in all cell lines tested. Overall, these results support our hypothesis that loss of HLA-I expression is regulated by epigenetic mechanisms in prostate cancer cells.

We also evaluated a panel of genes encoding antigen processing and presenting machinery (APM) proteins as well as beta-2-microglobulin (B2M) to determine whether DNMT and HDAC inhibition also increased expression of these genes. Expression of multiple APM genes, including TAP1/2 and B2M, has been demonstrated to be downregulated in prostate cancer ${ }^{24}$. Overall, inducibility of these genes was heterogenous and less robust than inducibility of HLA-I (Figure S5). Interestingly, TAP1 protein expression was induced by 5AZA2 in LNCaP cells and LBH in 22rv1 and PC3, as well as the combination treatment in all cell lines tested, suggesting epigenetic induction of APM may accompany HLA-I induction in certain cell lines. 
To confirm that the changes in gene and protein expression of HLA-I in response to DNMT and HDAC inhibitors are accompanied by epigenetic changes in the genes themselves, we measured DNA methylation and histone modification changes in response to SGI, 5AZA2, and LBH. HLA-I methylation was reduced across the HLA-I genes in response to SGI and 5AZA2 (Figure 4E,F), regardless of whether gene expression was significantly induced from SGI treatment alone, suggesting DNA methylation loss may not be sufficient in all cases to re-express HLA-I. This may be due to histone modifications not changing from a repressive state even when DNA methylation has been removed. This is supported by the retention of $\mathrm{H} 3 \mathrm{~K} 27 \mathrm{me} 3$ and H3K27ac levels in 22rv1 cells when treated with SGI (Figure S6A). This can also explain why cell lines tended to show the strongest response to combination treatments. To analyze changes in histone acetylation in response to HDAC inhibition by LBH, we performed ChIP analysis to look at H3K27ac in treated cell lines. H3K27ac was significantly increased in response to LBH in 22rv1 cells (Figure 4G) and a similar trend was observed in LNCaP and LAPC4 cells, though the results were not statistically significant.

Enrichment of RNA polymerase II (RPB1) at the HLA-I gene promoters was increased in LNCaP cells in response to SGI treatment, showing that the increase in gene expression is associated with increased transcriptional activity (Figure 4H). RBP1 enrichment at the HLA-I promoters was also increased in 22rv1 cells in response to LBH (Figure 4I). However, RPB1 enrichment was not increased in LNCaP cells in response to LBH treatment (Figure S6B). This difference in LBH induced RPB1 binding between LBH treated 22rv1 and LNCaP cells is corroborated by the differences in inducibility of HLA-I gene expression in response to LBH in these two cell lines. While responses to DNMT and HDAC inhibition were varied in prostate cancer cell lines, the changes to the epigenetic landscape that accompany gene and protein induction suggest modulation of epigenetic proteins in prostate cancer may be useful to reexpress epigenetically silenced HLA-I in patients.

\section{DNMT and HDAC Inhibition Induces HLA-I Gene Expression Ex Vivo}

The in vitro data suggest that DNMT and HDAC inhibition can significantly alter the expression of HLA-I. We next wanted to test whether this holds true in a more physiologically relevant system. Primary prostate tumor tissue was acquired from radical prostatectomy specimens and grown in an ex vivo culture. Expression of the HLA-I genes and three prostate specific genes, AR, KLK3 (PSA), and ACP3 (PAP), was measured to establish baseline HLA-I levels and confirm the presence of prostate cells in the culture (Figure 5A). Tissue in ex vivo culture was treated with DMSO, 5AZA2, or LBH either alone or in combination and induction of HLA-I expression was measured (Figure 5B). Similar to our in vitro cell culture studies, we found considerable heterogeneity regarding both the magnitude and pattern of HLA-I gene induction in 
response to either drug among these patient samples. 3 out of 5 patients showed robust induction in response to at least one of the treatment regimens. Among the responders, 2 out of 3 responded to 5AZA2 and showed no response to LBH alone. 1 out of 3 responders showed strong induction to $L B H$ and less response to 5AZA2. In 2 out of 5 patient samples we saw negligible response to 5AZA2 or LBH treatments. Induction of APM genes and B2M was also variable, but tended to correlate with HLA-I induction (Figure S7). Overall, these ex vivo culture studies corroborate the cell line data and indicate a biologically relevant role for DNMT and HDAC inhibition in re-expression of HLA-I in prostate cancer. Moreover, these data again highlight the variability in response to inhibition of these two classes of epigenetic regulators and underlines the multiple epigenetic mechanisms that are at play.

\section{HLA-I Upregulation on Tumor Cells by DNMT and HDAC Inhibition Enhances Activation of PSMA Specific T-cells}

We next sought to assess the functional relevance of HLA-I upregulation by DNMT and HDAC inhibition. To do this, we measured the $C D 8^{+} \mathrm{T}$-cell response to $\mathrm{LNCaP}$ cells treated with 5AZA2, SGI, and LBH alone and in combination. $\mathrm{PSMA}_{27-38-S p e c i f i c} \mathrm{CD}^{+}$ T-cells were raised by peptide vaccination in HHD mice expressing humanized HLA$A^{*} 02$. HLA-A*02 expressing LNCaP cells were pre-treated with $5 A Z A 2, S G I$, or LBH or a combination of $S \mathrm{SI}$ or $5 \mathrm{AZA} 2$ and $\mathrm{LBH}$ and then co-cultured with splenocytes from vaccinated or unvaccinated mice (Figure 6A). As an additional control, an anti-HLA-I blocking antibody was used to block HLA-I at the LNCaP cell surface. After co-culture, $\mathrm{T}$-cell activation markers were measured by flow cytometry in T-cells from each coculture treatment condition (Figure $6 \mathrm{~B}$ ). We found that $\mathrm{PSMA}_{27-38}$ tetramer-positive $\left(\mathrm{PSMA}^{+}\right) \mathrm{CD}^{+} \mathrm{T}$-cells that were co-cultured with LNCaP cells treated with any DNMT or HDAC inhibitor increased in frequency and expressed increased levels of activation markers CD69 and LFA-1 compared to those co-cultured with DMSO treated LNCaP cells. These T-cells also expressed increased levels of granzyme B and interferon- $\gamma$ (IFN- $\gamma$ ), markers indicative of T-cell stimulation and differentiation into cytotoxic T-cells (CTLs). Additionally, the population of T-cells expressing CD107 (LAMP1), a marker of $\mathrm{T}$-cell degranulation, was increased in the T-cells co-cultured with $\mathrm{LNCaP}$ cells treated with 5AZA2, SGI, and/or LBH.

The percent of PSMA ${ }^{+} \mathrm{CD} 8^{+}$T-cells present after co-culture with $\mathrm{LNCaP}$ cells in each treatment condition is shown in Figure $5 \mathrm{C}$. Treatment of $\mathrm{LNCaP}$ cells with $\mathrm{SGI}$ and $\mathrm{LBH}$ in combination significantly increased the percent of PSMA ${ }^{+} \mathrm{CD} 8^{+} \mathrm{T}$-cells after coculture with LNCaP cells at a 2:1 or 1:1 T-cell effector to tumor target (E:T) ratio SGI alone was able to significantly increase the percent of PSMA ${ }^{+} \mathrm{CD} 8^{+} \mathrm{T}$-cells after coculture with LNCaP cells at a 2:1 T-cell effector to tumor target ratio. The percent of $\mathrm{PSMA}^{+} \mathrm{CD}^{+}{ }^{+} \mathrm{T}$-cells present increased after co-culture with LNCaP cells treated with $5 A Z A 2$ and $L B H$ in combination, though this did not reach statistical significance. 
Treatment of LNCaP cells with HLA-I blocking antibody was able to ablate these effects. No significant change in the percent of PSMA ${ }^{+}$T-cells was seen when LNCaP cells were co-cultured with T-cells from unvaccinated mice. This study confirms a clear functional role for HLA-I induction by DNMT and HDAC inhibition and suggests the utility of HLA-I re-expression for vaccine-based immunotherapies that rely on functional MHC-I expression on tumor cells.

\section{HLA-I is Methylated in Prostate Cancer Circulating Tumor Cells with Low HLA-I Protein Expression}

Circulating tumor cells (CTCs), which are thought to have metastatic potential, offer a window into the biology of solid tumors and have been validated as prognostic biomarkers in prostate cancer ${ }^{34-36}$. Since HLA-I gene expression was significantly reduced in metastatic lesions compared to primary tumors, we wanted to determine whether HLA-I downregulation is conserved in CTCs as part of the metastatic process. Additionally, our in vitro and ex vivo data suggest patients have highly diverse responses to epigenetic therapy in the context of HLA-I re-expression and that only certain subsets of patients may benefit from specific therapies. Therefore, developing an epigenetic-based biomarker would be useful to determine which patients harbor epigenetic modifications in HLA-I and may benefit from epigenetic therapy.

We first characterized HLA-I protein expression in CTCs, as this has not yet been reported in the literature. We isolated prostate cancer CTCs from whole blood and stained with Hoechst and antibodies targeting cytokeratin (CK) to identify cells of epithelial origin, a panel of white blood cell (WBC) markers to identify white blood cells, and HLA-I. We gathered separate cohorts to stain CTCs for both extracellular HLA-I and intracellular HLA-I and compared the expression of HLA-I in individual CTCs to the median expression of HLA-I in the matched WBC populations (Figure 7A). We found that intracellular HLA-I expression in CTCs is heterogenous, but 7 out of 8 patients had at least one CTC with expression below the median WBC level of expression. Half of the patients had intracellular HLA-I expression below the median WBC level in all CTCs detected. For the extracellular expression cohort, all 8 samples had CTCs with extracellular expression of HLA-I below the level of median WBC expression and 7/8 patients had the majority of CTCs fall below the WBC median expression level. A representative image of a CTC and WBC is shown in Figure 7B.

We then evaluated whether CTCs with low HLA-I protein expression also harbored increased levels of HLA-I DNA methylation. To do this, we utilized a semiautomated single cell aspiration system to individually aspirate CTCs with high or low HLA-I protein expression ${ }^{37}$. EpCAM-positive CTCs were isolated and stained with Hoechst and antibodies to HLA-ABC, EpCAM, and markers of WBCs ${ }^{38,39}$. Stained cells were seeded onto a microwell array for aspiration (Figure S8A). CTCs were then aspirated based on relative levels of HLA-ABC binding determined by fluorescence 
microscopy for each patient sample. A representative example image of a cell in each of these populations is presented in Figure 7C. Groups of approximately 10-15 HLA-I positive or HLA-I negative CTCs were collected. We confirmed HLA-I expression differences in the identified populations by analyzing the mean fluorescent intensity (MFI) of HLA-ABC binding in each group (Figure 7D). HLA-I expression in the HLA-I negative CTC populations was significantly lower than the HLA-I positive CTC populations in all patients and significantly lower than matched WBC populations where available ( 3 out of 4 patients). Of note, HLA-I expression in the HLA-I positive CTC groups was lower than matched WBCs. Though the differences between the HLA-I positive CTC groups and WBCs were not statistically significant, this lower level of HLAI expression may be functionally significant and epigenetically regulated. A negative control group of 10 WBCs as well as a positive control group of $10 \mathrm{LNCaP}$ cells were collected by single cell aspiration for comparison.

Methylated DNA was enriched using the SEEMLIS (Semi-Automated ESP(Exclusion-Based-Sample-Preparation) Enrichment of Methylated-DNA from Low Input Samples) method of MBD2 protein-based enrichment of enzymatically digested DNA from the collected groups of CTCs and controls ${ }^{40}$. Enriched methylated DNA was subjected to qPCR with primers targeting HLA-I genes and LINE1, a control for successful enrichment of methylated DNA (Figure S8B). We were able to successfully detect HLA-I methylation from 10 aspirated LNCaP cells, while no methylation was detected in the WBC group (Figure 7E). LINE1 methylation was detected in all patient samples and controls (Figure 7E,F). We were able to detect HLA-I methylation in all four patient samples, though the pattern of detection and association with protein expression varied (Figure 7G, S8C).

Patient 568, 588, and 490 had higher methylation in the HLA-I genes in the HLA-I protein negative CTC populations than the HLA-I protein positive CTC populations. Levels of methylation were between $50 \%$ and $100 \%$ of the level of methylation in the LNCaP control sample for HLA-A and HLA-B, when detected, in these patients. HLA-C methylation levels were lower, but present in the HLA-I protein negative populations in patient 490 . In contrast, patient 487 had detectable HLA-I methylation in both groups of CTCs, with higher levels detected in the HLA-I positive population. HLA-A was also detected in the HLA-I protein positive populations at low levels in patient 568 and 588 and at higher levels in patient 490. As mentioned above and shown in Figure 7D, the HLA-I expression level in the HLA-I positive CTC groups tended to be lower than the matched WBC expression, suggesting that low, non-negative HLA-I expression may still be epigenetically regulated in some patients. These patients may still benefit from epigenetic therapy to express HLA-I to levels closer to that of WBCs. Overall, this experiment represents the first analysis of HLA-I methylation in prostate cancer CTCs and provides a foundation for future biomarker studies. 


\section{DISCUSSION}

The evidence presented here demonstrates that epigenetic mechanisms regulate expression of HLA-I genes in human prostate cancer. While it has been known for decades that HLA-I is downregulated at the protein level in prostate cancer, there have been limited investigations into the molecular underpinnings of this phenomenon, especially as it relates to epigenetic regulation of these genes. To address this lack of understanding, we have described the HLA-I methylome in prostate cancer patient samples and cell lines and confirmed the presence of repressive histone modifications in cell line models. We have also for the first time measured HLA-I protein expression in circulating tumor cells (CTCs) from patients with prostate cancer and found that low HLA-I expression in CTCs was associated with increased levels of HLA-I DNA methylation.

Our investigations into the regulation of class I HLA genes in prostate cancer revealed frequent downregulation of HLA-I gene expression in metastatic tumors. The striking decrease in HLA-I expression and the presence of HLA-A DNA methylation signatures in metastatic lesions identified in this study implies a possible role for epigenetic loss of HLA-I expression in progression to metastasis. This idea is supported by previous findings showing that promoter DNA methylation increases during progression and that epigenetic mechanisms are important drivers of prostate cancer progression ${ }^{41-43}$. Whether the epigenetic alterations in HLA-I genes are a driver or passenger in the metastatic cascade will need to be further studied, but our data suggests that immune evasion through epigenetic downregulation of HLA-I is a frequent event in metastatic prostate cancer.

This study is in accordance with previous work showing HDAC inhibition upregulates HLA-I gene and proteins levels in LNCaP cells ${ }^{14}$, and gene expression levels in PC3 and Du145 cells ${ }^{14,44}$. Our work expands on these studies by examining the basal epigenetic signatures as well as changes in those signatures in response to HDAC and DNMT inhibition in multiple cell lines. We strengthen this idea further by showing biological relevance for HLA-I induction by DNMT and HDAC inhibition in ex vivo tissue samples. Importantly, we also showed that HLA-I gene and protein expression can be functionally induced on tumor cells by DNMT and HDAC inhibition, leading to increased activation of co-cultured T-cells from mice vaccinated with PSMApeptide. This study demonstrated the potential power of combining therapeutic reexpression of epigenetically silenced HLA-I with an MHC-I dependent immunotherapy.

A previous study from our lab found DNMT and/or HDAC inhibition induces expression of CTAs ${ }^{38}$ and another study found APM molecules were upregulated with HDAC inhibition ${ }^{44}$. Our current study supports a wider role for epigenetics in regulating antigen presentation by also downregulating the HLA-I genes themselves. Inhibition of DNMT and HDACs likely affects many cellular pathways in addition to HLA-I genes, 
CTAs, and APM, leading to the phenotypes we observed in this study. In line with this, our ex vivo gene expression analysis suggests a role for APM and B2M epigenetic regulation in prostate cancer patients, which merits further study. A recent study found inhibition of BET bromodomain containing proteins, which are readers of histone acetylation, led to increased HLA-I protein expression and immunogenicity in vivo, supporting the important role we have found for histone modifications in HLA-I regulation ${ }^{45}$. Further investigation into the contributions of other affected cellular pathways is needed to fully understand this phenomenon.

DNMT and HDAC inhibitors have been explored for their possible therapeutic efficacy and numerous clinical trials are ongoing for single or combination uses, including trials with $5 \mathrm{AZA} 2, \mathrm{SGI}$, and $\mathrm{LBH}^{46}$. Recently, the first epigenetic therapy for solid tumors, a small molecule drug targeting EZH2, was approved by the FDA for use in epithelioid carcinoma ${ }^{47,48}$. Previously, the only FDA approved uses for drugs targeting epigenetic modifying proteins were for hematologic malignancies. The limited success of epigenetic therapies in solid cancers may be due to the heterogeneity in the epigenetic signatures and responses to therapies as evidenced in this study ${ }^{21}$. Additionally, failure of immunotherapies has been attributed to lack of immunogenicity of tumor cells as well as the inability to monitor treatment response in solid tumors ${ }^{22}$. We anticipate that the ability to identify patients likely to respond to therapeutic reexpression of HLA-I and combination immunotherapy by monitoring epigenetic signatures in circulation may alleviate some of these challenges. This study is the first to measure HLA-I expression and matched methylation in CTCs. Further investigation into how HLA-I methylation in circulation can inform on treatment options or outcomes is certainly warranted and is a goal of our future studies. Overall, this study has implicated epigenetic mechanisms in the regulation of HLA-I in prostate cancer and points to epigenetic therapy as a promising option for enhancing the immune response in prostate tumors.

\section{METHODS}

\section{Analysis of Public Data Sets}

Genomic alteration data was accessed and analyzed in cBioPortal (RRID: SCR_014555) ${ }^{49,50}$. TCGA-PRAD data was accessed and downloaded through UCSC Xena ${ }^{51}$. Data from Taylor, et. al. ${ }^{25}$ was accessed, analyzed, and downloaded through cBioPortal. ATAC-seq data from Corces, et al. was accessed, analyzed, and downloaded through UCSC Xena ${ }^{51,52}$. Methylation beta values and matched gene expression values were accessed through Wanderer ${ }^{53}$. Probe information is listed in Table S4. Prism 8 (GraphPad Prism, RRID: SCR_002798) was used for correlation analyses. Z-scores for gene expression and ATAC-seq were calculated with the following formula: 


$$
Z=\frac{\chi-\mu}{\sigma}
$$

where $\chi$ is the tumor or metastasis gene expression value, $\mu$ is the normal sample population mean, and $\sigma$ is the normal sample population standard deviation. In the ATAC-seq data set, z-scores were calculated as compared to the tumor population, where $\chi$ is the tumor gene or ATAC-seq expression value, $\mu$ is the tumor sample population mean, and $\sigma$ is the tumor sample population standard deviation.

\section{COMPARE-MS}

Patient samples used in this analysis were previously described ${ }^{26,29}$. COMPARE-MS was performed as previously described ${ }^{26,27,29}$. Briefly, DNA was digested with Alul and Hhal restriction enzymes (New England Biolabs, Cat\# R0137L and Cat\# R0139L) and enriched with MBD2-MBD protein (Takara Bio, Cat\# 631962). Enriched DNA was subjected to qPCR with primers targeting HLA-A (Fwd: TCTGCGGGGAGAAGCAAG; Rev: GGGACACGGATGTGAAGAAA). Methylation index was calculated by normalizing Ct values from samples to controls (enzymatically methylated white blood cell DNA) to generate a Methylation Index from a range of 0.0-1.0.

\section{Cell Lines and Cell Culture} LAPC4 (ATCC, Cat\# CRL-13009, RRID: CVCL_4744) were maintained in DMEM Medium (Corning) supplemented with $20 \%$ fetal bovine serum (FBS) (Gibco, Cat\# 10437028), 1\% sodium pyruvate (Corning, Cat\# MT25000Cl), 0.5\% betamercaptoethanol, and 1\% penicillin-streptomycin (HyClone, Cat\# SV30010). LAPC4 cells were cultured in poly-d-lysine coated flasks and/or plates (BioCoat flasks: Corning, Cat\# 0877260; 6-well plates: Sigma-Aldrich, Cat\# Z720798-20EA). RWPE1 (ATCC Cat\# CRL-11609, RRID: CVCL_3791, LNCaP (ATCC, Cat\# CRL-1740, RRID: CVCL_1379), 22Rv1 (ATCC, Cat\# CRL-2505, RRID: CVCL_1045), and PC3 (ATCC, Cat\# CRL-1435, RRID: CVCL_0035) cells were maintained in RPMI 1640 Medium (Corning, Cat\# MT10040CV) supplemented with 10\% FBS, 1\% sodium pyruvate, $1 \%$ penicillin-streptomycin, 1\% non-essential amino acids (HyClone, Cat\# SH30238.01), and $0.1 \%$ beta-mercaptoethanol. LCL (HCC2218-BL, ATCC, Cat\# CRL-2363, RRID: CVCL_1264) cells were grown in suspension in RPMI 1640 Medium supplemented with $10 \%$ FBS and 1\% penicillin-streptomycin. LCL, RWPE1, LNCaP, 22rv1, and PC3 were cultured in tissue culture treated flasks and/or plates (Flasks: Corning, Cat\# 07202000; Plates: Thermo Fisher Scientific, Cat\# 087721G). All cell lines were used in experiments within 30 passages from thaw. RWPE1, LNCaP, PC3, and LAPC4 were authenticated by short tandem repeat and tested for mycoplasma by PCR in 2017.

\section{Ex Vivo Culture of Prostate Tissue}

Human prostate tissues were obtained from patients undergoing radical prostatectomy at the University of Wisconsin-Madison. All patients were consented under an 
Institutional Review Board (IRB) protocol \#20130653. Absorbable gelatin sponges (Ethicon, Cat\# 1973) were cut into pieces to fit in a 24-well tissue culture plate. Sponges were soaked in Ham's F-12 media (Fisher Scientific, Cat\# SH3002601) supplemented with 0.25 units $/ \mathrm{ml}$ regular insulin (Sigma-Aldrich, Cat\# 19278-5ML), $1 \mu \mathrm{g} / \mathrm{mL}$ hydrocortisone (Sigma-Aldrich, Cat\# H0888-1g), $5 \mu \mathrm{g} / \mathrm{mL}$ human transferrin (SigmaAldrich, Cat\# T8158-100mg), $2.7 \mathrm{mg} / \mathrm{ml}$ dextrose, $0.1 \mathrm{nM}$ non-essential amino acids (HyClone, Cat\# SH30238.01), 100 units $/ \mathrm{ml}$ and $100 \mu \mathrm{g} / \mathrm{mL}$ Penicillin/Streptomycin respectively (HyClone, Cat\# SV30010), 2 mM L-glutamine (Corning, Cat\# 25-005-Cl), $25 \mu \mathrm{g} / \mathrm{mL}$ bovine pituitary extract (Life Technologies, Cat\# 13028014), and $1 \%$ fetal bovine serum (FBS) (Gibco, Cat\# 10437028) until fully saturated. Each core was cut

into $\sim 1 \mathrm{~mm}^{2}$ by $1 \mathrm{~mm}^{2}$ cubes. Tissue was placed on the sponges and cultured for up to 4 days at $37^{\circ} \mathrm{C}$ at $5 \% \mathrm{CO}_{2}$ and $500 \mu \mathrm{L}$ media was replaced daily.

\section{Immunoblotting}

Whole cell lysates were collected from adherent cells by scraping into RIPA buffer after washing with cold PBS. Whole cell lysates were separated by SDS-PAGE and transferred onto nitrocellulose membrane. Membranes were blocked with SuperBlock blocking buffer (Thermo Scientific, Cat\# 37515). Membranes were probed with primary antibodies diluted in $3 \%$ BSA in TBS plus $0.1 \%$ Tween- 20 at $4^{\circ} \mathrm{C}$ overnight followed by incubation with HRP-linked secondary antibody (BioLegend, Cat\# 405306, RRID:AB_315009) at RT for 1 hour and visualization by chemiluminescence. Primary antibodies: HLA-I clone W6/32 (BioLegend, Cat\# 311412, RRID:AB_493132), $\alpha$-tubulin (BioLegend, Cat\# 627901, RRID:AB_439760).

\section{Flow Cytometry Analysis of Cell Lines}

Cells were stained with Ghost Dye ${ }^{\mathrm{TM}}$ Violet 510 (Tonbo Biosciences, Cat\# 13-0870) to identify viable cells and PE-Cy7 conjugated anti-HLA-ABC antibody (BioLegend, Cat\# 311430, RRID:AB_2561617). Intracellular staining for PSMB8 (LMP7)-PE (Abcam, Cat\#EPR14482) Calreticulin-AlexaFluor647 (MBL International, Cat\#K0136-4; RRID:AB_592808) and TAP-1-FITC (Abcam, Cat\#EPR3924; RRID:AB_2819061) protein expression was performed following manufacturer's protocol with $B D$ Cytofix/Cytoperm kit (BD Biosciences, Cat\#554714; RRID:AB_2869008). Samples were acquired on an LSR II instrument and data analyzed by the FlowJo software v9.9.6 (FlowJo, RRID: SCR_008520). Median Fluorescent Intensity was analyzed on gated live, single cells.

\section{MBD2-MBD Enrichment of Methylated DNA from Cell Lines}

Genomic DNA was isolated from cells using the AllPrep RNA/DNA Mini Kit (Qiagen, Cat\# 80204) according to manufacturer's instructions. DNA was quantified by a NanoDrop 1000 spectrophotometer and $1 \mu \mathrm{g}$ DNA was sheared by sonication to an 
average size of around 200bp. Methylated DNA was enriched from sheared genomic DNA using the EpiXplore Methylated DNA Enrichment Kit (Takara Bio, Cat\# 631962) according to manufacturer's instructions. Enrichment was measured by qRT-PCR using primers designed to various regions of HLA-A, HLA-B, and HLA-C (Integrated DNA Technologies). Primers are listed in Table S5.

\section{Chromatin Immunoprecipitation}

Chromatin immunoprecipitation (ChIP) was performed according to manufacturer's instructions using the SimpleChIP Enzymatic Chromatin IP Kit with Magnetic Beads (Cell Signaling Technology, Cat\# 9003S). Immunoprecipitation was performed using the following antibodies from Cell Signaling Technology: Histone H3 (Clone D2B12, Cat\# 4620S, RRID: AB_1904005), H3K27ac (Clone D5E4, Cat\# 8173S, RRID:

AB_10949503), H3K27me3 (Clone C36B11, Cat\# 9733S, RRID: AB_2616029), and IgG (Cat\# 2729S, RRID: AB_1031062). DNA was then analyzed by qPCR using primers designed to target $H L A-A, H L A-B$, and HLA-C (Integrated DNA Technologies). Primers are listed in Table S5. The H3K27ac signature in GM12878 determined by ChIP-seq from the ENCODE consortium ${ }^{33}$ was accessed in the UCSC Genome Browser (RRID: SCR_005780) to aid in primer design.

Epigenetic Drug Treatments of Cell Lines and Ex Vivo Tissue 5-Aza-2'-deoxycytidine (5AZA2) (Sigma-Adrich, Cat\# A3656-5MG), Panobinostat (LBH, LBH589) (Selleckchem, Cat\# S1030), and SGI-110 (SGI) (Astex Pharmaceuticals) were dissolved in DMSO and stored at $-80^{\circ} \mathrm{C}$ in aliquots. Cells were treated with $10 \mu \mathrm{M}$ $5 A Z A 2,1 \mu \mathrm{M} \mathrm{SGI}$, or DMSO for 72 hours. $10 \mathrm{nM}$ or $100 \mathrm{nM} \mathrm{LBH}$ was added for the last 24 hours after 48 hours of $5 A Z A 2$ or SGI treatment for combination treatments.

Gene Expression Analysis in Cell Lines

Total RNA was isolated from cells using the AllPrep RNA/DNA Mini Kit (Qiagen, Cat\# 80204 ) according to manufacturer's instructions. RNA was quantified by a NanoDrop 1000 spectrophotometer and $1 \mu \mathrm{g}$ total RNA was reverse transcribed using the High Capacity RNA-to-cDNA kit (Thermo Fisher Scientific, Cat\# 4388950). cDNA was diluted 10x and $5 \mathrm{uL}$ was used per reaction for qPCR. Pre-designed TaqMan probes (Thermo Fisher) for HLA-A (Hs01058806_g1), HLA-B (Hs00818803_g1), and HLA-C (Hs00740298), and RPLPO (4333761F) were used with iTaq Universal Probes Supermix (BioRad, Cat\# 1725135). Gene expression was determined using the deltadelta-Ct method after normalization of each gene to housekeeping gene, RPLPO (PO).

Gene Expression Analysis in Epigenetic Drug Treated Ex Vivo Tissue and 5AZA2/LBH Treated Cell Lines 
Total RNA was isolated from cells using RNeasy Mini Kit (Qiagen, Cat\# 74106) according to manufacturer's protocol. Total RNA was isolated from ex vivo tissue using the Aurum ${ }^{\text {TM }}$ Total RNA Fatty and Fibrous Tissue Kit (BioRad, Cat\# 7326830) according to the manufacturer's protocol. RNA was quantified by a NanoDrop 1000 spectrophotometer and $1 \mu \mathrm{g}$ total RNA was reverse transcribed using iScript Reverse Transcription Supermix (Bio-Rad, Cat\# 1708841). 1 $\mu \mathrm{L}$ of the cDNA synthesis reaction was used to perform qPCR using SsoAdvanced Universal SYBR Green Supermix (BioRad, Cat\# 1725274) according to the manufacturer's protocol. HLA-A, HLA-B, HLA$C$, and RPLPO primers are listed in Table S5. Gene expression was determined using the delta-delta-Ct method after normalization of each gene to housekeeping gene, RPLPO (PO).

Peptide Vaccinations and T-cell Co-Culture PSMA specific $C D 8^{+} \mathrm{T}$-cells were generated by $\mathrm{PSMA}_{27-38}$ peptide vaccination of $\mathrm{HHD}$ transgenic humanized mice expressing human HLA-I $A^{*} 02$. The HHD mice were a generous gift from Professor Francois Lemonnier at the Pasteur Institute, Paris ${ }^{54}$. Mice were given once weekly subcutaneous injections of $100 \mathrm{ug}$ synthetic PSMA peptide (VLAGGFFLL) (Prolmmune, Oxford, UK) in 100uL CFA (Thermo Fisher Scientific, Cat\# NC0916022) for the first injection or IFA vehicle (Sigma-Aldrich, Cat\# AR002) for subsequent injections. Splenocytes were harvested 1 week after last immunization and the number of live $\mathrm{PSMA}_{27-38}$-specific $\mathrm{CD}^{+}$splenocytes was determined by flow cytometry analysis following staining with GhostDye ${ }^{\mathrm{TM}}$ Violet 510 (Tonbo Biosciences, Cat\# 13-0870), anti-mouse CD8 antibody (Tonbo Biosciences, Cat\# 25-0081, RRID:AB_2621623) and Pro5 ${ }^{\circledR}$ PSMA 27-38 A*02:01 MHC I pentamer (Prolmmune, Oxford, UK). PSMA vaccinated splenocytes were then co-cultured with LNCaP cells that were pretreated with DMSO vehicle or 10uM 5AZA2 or $1 \mathrm{UM} \mathrm{SGI}$ for 72 hours and/or $10 \mathrm{nM}$ LBH for the last 24 hours in RPMI media supplemented with $10 \% \mathrm{FBS}$. In control co-culture wells, LNCaP cells were treated with $5 \mu \mathrm{g}$ of purified anti-HLA-A,B,C blocking antibody (clone W6/32) (BioLegend, Cat\# 311412, RRID:AB_493132) prior to adding splenocytes. Cells were co-cultured for 72 hours and Golgi-stop (BD Biosciences, Cat\# 554724 ) was added for the last 4 hours of culture, following the manufacturer's protocol. Cells were then harvested and subjected to labeling with Ghost Dye ${ }^{\mathrm{TM}}$ Violet 510 and surface markers: CD8 (Tonbo Biosciences, Cat\# 25-0081, RRID:AB_2621623), Pro5 ${ }^{\circledR}$ $\mathrm{PSMA}_{27-38} \mathrm{~A}^{*}$ 02:01 MHC I pentamer (Prolmmune), CD69 (BD Pharmigen, Cat\# 551113, RRID:AB_394051), LFA-I (BD Biosciences, Cat\# 558191, RRID:AB_397055), CD107 (BD Biosciences, Cat\# 564347, RRID:AB_2738760) and CD16/CD32 Fc Block 2.4G2 (BD Biosciences Cat\#553142) followed by fixation and permeabilization with BD Cytofix/Cytoperm (Thermo Fisher Scientific, Cat\# 554714) according to the manufacturer's protocol and intracellular staining with antibodies against murine IFN $\gamma$ (Tonbo Biosciences, Cat\# 20-7311, RRID:AB_2621616) and Granzyme B (BD 
Biosciences, Cat\# 560211, RRID:AB_1645488). Cells were then washed and acquired on an LSR II Fortessa or an Attune NxT instrument followed by data analysis by the FlowJo software v9.9.6 (FlowJo, RRID: SCR_008520). Gating controls included the fluorescent minus one (FMO) strategy.

CTC Capture, Imaging, and Analysis

Blood samples were collected from patients after receiving written informed consent under a protocol approved by the IRB at the University of Wisconsin-Madison. CTCs were processed and stained as previously described in Sperger et. al ${ }^{38}$. Briefly, PBMCs were isolated from whole blood on Ficoll-Paque PLUS (GE Healthcare, Cat\# 45-001750) gradient and fixed with Cytofix Fixation Buffer (BD Biosciences, Cat\# 554655). Fixed cells were incubated with paramagnetic particles (PMPs) (Dynabeads ${ }^{\circledR}$ FlowComp ${ }^{\mathrm{TM}}$ Flexi kit, Life Technologies, Cat\# 11061D) coated with biotinylated antiEpCAM antibody (R\&D Systems, Cat\# AF960, RRID: AB_355745). The Versatile Exclusion-based Rare Sample Analysis (VERSA) platform was used for enrichment and staining of CTCs, as described previously ${ }^{38,55,56}$. PMP bound cells were isolated in the VERSA and stained with Hoechst 33342 (Thermo Fisher, Cat\# PI62249) and antibodies to the proteins indicated in the corresponding figures, which are summarized in Table S6. Pan-cytokeratin (CK) was conjugated to Alexa Fluor ${ }^{\mathrm{TM}} 790$ using the Alexa Fluor ${ }^{\mathrm{TM}}$ 790 Antibody Labeling Kit (Life Technologies, Cat\# A20189) according to manufacturer's instructions. All other antibodies were purchased pre-conjugated to the fluorophores listed in Table S6. CD45, CD34, and CD11b were used on the same channel to serve as a white blood cell (WBC) "exclusion channel". CD14 and CD27 were included in addition to CD45, CD34, and CD11b in the WBC exclusion channel for the experiment measuring extracellular HLA-I in CTCs only. Cells were stained for extracellular markers at $4^{\circ} \mathrm{C}$ for 30 minutes. Cells were permeabilized and stained for intracellular antibodies with $\mathrm{BD}$ Perm $/$ Wash at $4^{\circ} \mathrm{C}$ overnight (BD Biosciences, Cat\# BDB554723). Cells were imaged in the VERSA at 10x magnification using NIS Elements AR Microscope Imaging Software (NIS-Elements, RRID:SCR_014329) and analyzed using NIS Elements analysis software. CTCs were defined as positive for Hoechst and CK and negative for CD45/34/11b or CD45/34/11b/14/27. All other cells were considered part of the WBC population.

\section{Single Cell Aspiration of CTCs for Methylation Analysis}

CTCs enriched using the VERSA platform as described above were stained in the VERSA with Hoechst 33342 (Thermo Fisher, Cat\# PI62249) and anti-bodies to HLA-I, EpCAM, CD27, CD45, CD34, and CD11b. Fluorophores, catalog numbers, and other antibody information is summarized in Table S6. Patient characteristics for single cell aspiration CTC samples are summarized in Table S7. Cells were then further enriched using a single cell aspiration platform, SASCA, as described by Tokar et al ${ }^{37}$. Briefly, 
cells were seeded into polydimethylsiloxane (PDMS) microwells mounted on a glass microscope slide. The microwell array was imaged on a Nikon Ti-E Eclipse inverted fluorescent microscope, and target cells were identified by phenotypic staining analysis. CTCs were identified as EpCAM positive, exclusion (CD45/CD34/CD11b/CD27) negative cells, whereas WBCs were classified as EpCAM negative, exclusion positive cells. CTCs were further subdivided into groups based on HLA-I positivity compared to WBCs in the same sample. Target cells were aspirated from microwells and dispensed directly into a droplet of PBS in the EXTRACTMAN extraction plate (Gilson, Cat\# 22100008) for DNA extraction. Images of the microarray were analyzed using NIS Elements AR Microscope Imaging Software (NIS-Elements, RRID:SCR_014329) to obtain HLA-I mean fluorescent intensity (MFI) values.

\section{DNA Extraction from CTCs}

Semi-automated DNA extraction was performed on a Gilson PIPETMAX liquid handling robot enabled for exclusion-based sample preparation (ESP), termed EXTRACTMAX ${ }^{57}$. LiDS buffer (90mM Tris-HCL, 500mM lithium chloride, 1\% Igepal CA-630, 10mM EDTA, $1 \mathrm{mM}$ dithiothreitol) and MagneSil Paramagnetic Particles (PMPs) (Promega, Cat\# MD1441) resuspended in GTC buffer $(10 \mathrm{mM}$ Tris-HCl, $6 \mathrm{M}$ guanidinium thiocyanate, $0.1 \%$ Igepal CA-630, pH 7.5) are added to the extraction microplate (Gilson, Cat\# 22100008) by the robot. Cells were added to the microplate well containing LiDS, GTC, and MagneSil beads and mixed by the robot. Cells were allowed to lyse and DNA was allowed to bind to MagneSil PMPs for 5 minutes. The robot then transferred the MagneSil PMPs with bound DNA by exclusion liquid repellency (ELR) through one PBST (PBS containing $0.1 \%$ Tween-20) wash, one PBS wash, and into $15 \mu \mathrm{L}$ of nuclease-free water (Promega, Cat\# P1197) for elution. Beads were manually resuspended in the elution well and allowed to elute for 2 minutes. The MagneSil PMPs are magnetically transferred out of the elution well, leaving eluted DNA in water.

\section{MBD2-MBD Enrichment of Methylated DNA from CTCs}

The SEEMLIS method of MBD2-MBD enrichment was performed ${ }^{40} .25 \mu \mathrm{L}$ of TALON magnetic beads (Takara, Cat\# 635637) were washed 3x with $100 \mu \mathrm{L} 1 \times$ Binding Buffer (BB) (4\% glycerol, $1 \mathrm{mM} \mathrm{MgCl}$, $0.5 \mathrm{mM}$ EDTA, $120 \mathrm{mM} \mathrm{NaCl}, 2 \mathrm{mM}$ Tris-HCl pH 7.4, $0.2 \%$ Tween-20, and $0.5 \mathrm{mM}$ DTT). Washed beads were resuspended in $100 \mu \mathrm{L}$ MBD2-

MBD Coupling Buffer (1x BB, 1x Halt protease inhibitor cocktail (Thermo Scientific, Cat\# PI87786), 500 ng Unmethylated Lambda DNA (Promega, Cat\# D1521), $5 \mu \mathrm{L}$ tagged MBD2-MBD (EpiXplore Kit, Takara, Cat\# 631962) and placed on shaker at RT for 1 hour to bind MBD2-MBD to the TALON beads. DNA was digested using $1 \mu \mathrm{L}$ of each restriction enzyme Alul at 10 units/ $\mu \mathrm{L}$ (New England Biolabs, Cat\# R0137L) and $\mathrm{HpyCH} 4 \mathrm{~V}$ at 5 units $/ \mu \mathrm{L}$ (New England Biolabs, Cat\# R0620L)) in $20 \mu \mathrm{L}$ reactions containing 1x CutSmart Buffer (New England Biolabs, Cat\# B7204S) for 15 minutes at 
$37^{\circ} \mathrm{C}$ followed by enzyme inactivation for 20 minutes at $80^{\circ} \mathrm{C}$. MBD2-MBD bound beads were washed $3 x$ with $100 \mu \mathrm{L} 1 \mathrm{x}$ BB and resuspended in $88 \mu \mathrm{L} 1 \mathrm{x}$ BB with 1x Halt protease inhibitor cocktail and added to $20 \mu \mathrm{L}$ restriction enzyme digested DNA in 200 $\mu \mathrm{L}$ PCR tubes. This reaction was placed on a shaker at RT for 3 hours to bind methylated DNA to MBD2-MBD conjugated TALON beads. PCR tubes were placed onto the Gilson PIPETMAX liquid handling robot (EXTRACTMAN system enabled for ESP as previously described ${ }^{57}$ ) for washing and elution steps. The robot transferred the whole volume from the PCR tubes onto the extraction microplate (Gilson, Cat\# 22100008) and then magnetically transferred the TALON beads through a wash containing $1 \times$ BB with 1x Halt protease inhibitor cocktail and into $15 \mu \mathrm{L}$ of water for elution. The whole elution volume including beads was manually pipetted into new 200 $\mu \mathrm{L}$ PCR tubes containing pre-amplification reaction mix and placed directly into the thermocycler under pre-amplification cycling conditions. Volumes indicated are per reaction.

Pre-amplification and qPCR of MBD2-MBD Enriched DNA from CTCs.

Quantitative PCR was performed using custom TaqMan hydrolysis probes (Applied Biosystems) and iTaq Universal Probes Supermix (Bio-Rad, Cat\# 1725153). Primer and probe sequences are listed in Table S5. Cycling conditions: 5 minutes at $95^{\circ} \mathrm{C}$ for initial denaturation and enzyme activation followed by 45 amplification cycles of 5 seconds at $95^{\circ} \mathrm{C}$ and 30 seconds at $60^{\circ} \mathrm{C}$. Pre-amplification was performed using the custom hydrolysis probes and TaqMan PreAmp Master Mix (Applied Biosystems, Cat\# 4488593) when indicated according to manufacturer specifications. Cycling conditions: 10 minutes at $95^{\circ} \mathrm{C}$ for enzyme activation followed by 14 cycles of $95^{\circ} \mathrm{C}$ for 15 seconds and $60^{\circ} \mathrm{C}$ for 4 minutes. Pre-amplified samples were diluted 1:5 with TE buffer. $\mathrm{Ct}$ values were transformed into relative methylation values by the following equation:

$$
\text { Relative Methylation }=2^{-(C t-M C V)}
$$

Where MCV is the max cycle value, which is the Ct cut off pre-determined by cell line validation studies for each gene. For HLA-I, MCV is equal to 33. For LINE1, MCV is equal to 45. A Methylation Index from 0.0-1.0 was also calculated for each patient using $\mathrm{LNCaP}$ methylation as 1.0 on the Methylation Index scale. Raw $\mathrm{Ct}$ values were converted to relative values using the delta $\mathrm{Ct}$ method and then divided by the relative methylation in LNCaP cells as follows:

$$
\text { Methylation Index }=\frac{2^{-C t_{C T C}}}{2^{-C t_{L N C a P}}}
$$

Statistical Analysis

Survival curve analysis was performed using the log-rank (Mantel-Cox) test. For the HLA-I genomic alteration and HLA-I protein expression studies, comparison between groups was made with an ordinary one-way ANOVA followed by post hoc analysis with 
the Tukey test for correction of multiple comparisons. Comparisons between average methylation beta values in tumor vs. normal samples from the PRAD data set were made by unpaired t-test with Welch's correction. Comparisons between methylation beta values across gene expression sub groups were made with an ordinary one-way ANOVA followed by post hoc analysis with the Tukey test for correction of multiple comparisons. For baseline gene expression and ChIP experiments, comparisons between groups were made with two-way ANOVA using the Dunnett method for correction of multiple comparisons. For drug treatment gene expression experiments, comparison between DMSO and treatment groups were made by t-test corrected for multiple comparisons by the Holm-Sidak method. For CTC MFI experiments, comparisons were made by Kruskal-Wallis test using the Dunn's method for correction of multiple comparisons. Gene expression statistical analyses were performed on deltaCt values. All statistical analyses were performed in Prism 8 (GraphPad Prism, RRID: SCR_002798).

\section{AUTHOR CONTRIBUTIONS}

T.S.R. and E.H. wrote and edited the manuscript, designed and conducted experiments, and analyzed data. All other authors reviewed and/or edited the manuscript. C.N.S., C.S.G., K.C., M.R.K., A.S., and T.E.G.K. conducted experiments. D.J.B. acquired funding, designed technology used in experiments. D.F.J. advised on study oversight and design and provided clinical samples. D.G.M. advised on study oversight and design and provided materials for animal studies. M.C.H. designed and conducted experiments, analyzed data, acquired funding, and advised on study oversight and design. J.M.L. advised on study oversight and design, acquired funding, and provided clinical samples.

\section{ACKNOWLEDGMENTS}

This work was supported by the Office of the Assistant Secretary of Defense for Health Affairs through the Prostate Cancer Research Program [W81XWH-17-1-0096 to T.S.R.] and [W81XWH-12-1-0052 to J.M.L], the National Cancer Institute of the National Institutes of Health [1R01CA247479-01 to D.J.B. and J.M.L], and a Movember Foundation - Prostate Cancer Foundation Award [17CHAL05 to J.M.L.]. M.C.H. is supported by a grant from the Safeway Foundation. The content is solely the responsibility of the authors and does not necessarily represent the official views of the above mentioned organizations. We would like to thank the patients who donated samples for this study. The results using TCGA-PRAD data sets are based on data generated by the TCGA Research Network: https://www.cancer.gov.tcga. We thank Dr. 
Bing Yang, clinical research coordinators at UW Urology, and staff as the UWCCC Translational Science Biocore (TSB) for assistance in patient consenting and donor sample acquisition. Additionally we acknowledge NIH grant support for UWCCC Flow Cytometry and TSB BioBank core services awarded to the University of Wisconsin Carbone Cancer Center (UWCCC) in Support Grant P30 CA014520 and NIH funding for the LSR Fortessa instrument \#1S100OD018202-01. 


\section{FIGURES}

Figure 1

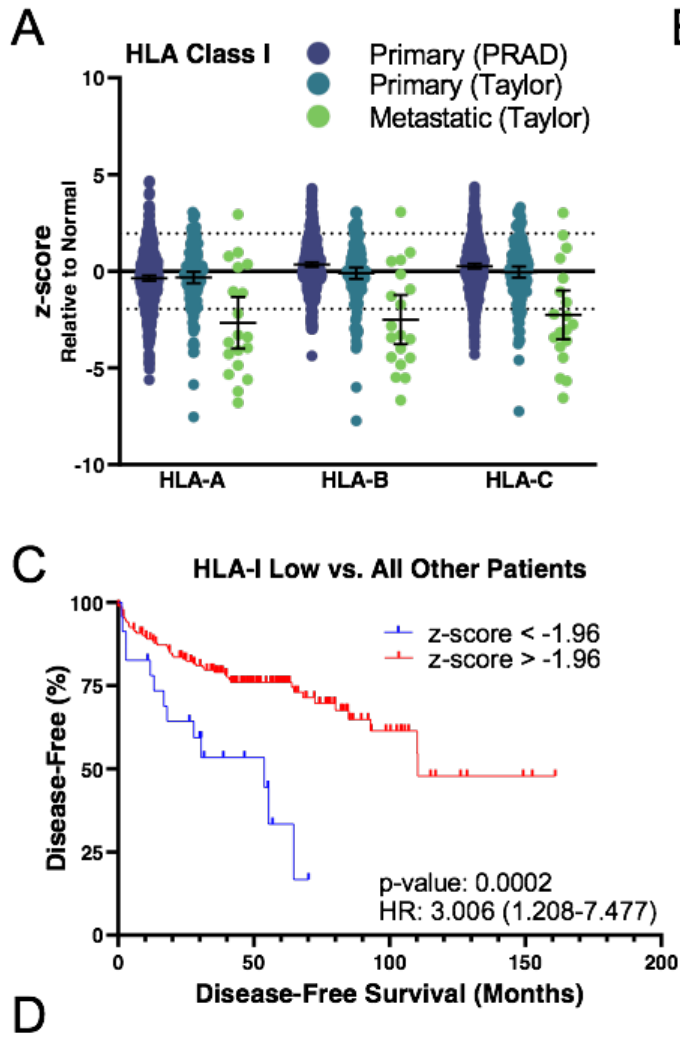

B

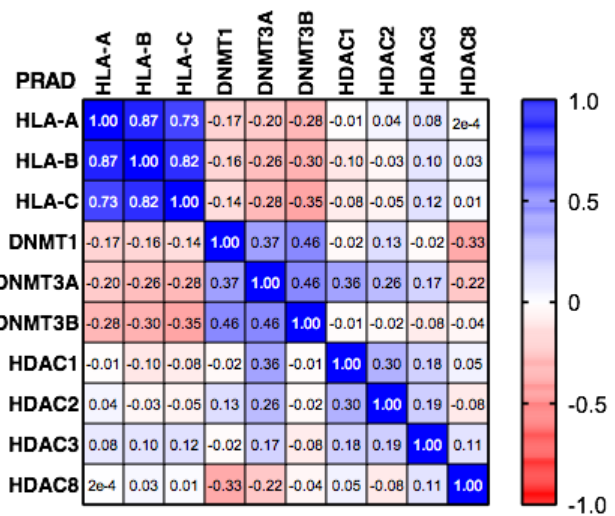

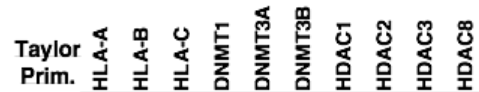

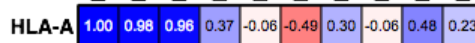

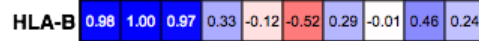

\begin{tabular}{llllllllllllll} 
HLA-C & 0.96 & 0.97 & 1.00 & 0.32 & -0.08 & -0.50 & 0.33 & -0.10 & 0.53 & 0.21 \\
\hline
\end{tabular}

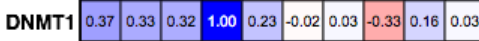

\begin{tabular}{ll|llllllllll} 
DNMT3A & -0.06 & -0.12 & -0.08 & 0.23 & 1.00 & 0.40 & 0.11 & -0.37 & 0.08 & -0.21 \\
\hline
\end{tabular}

\begin{tabular}{llllllllllll}
\hline DNMT3B & -0.49 & -0.52 & -0.50 & -0.02 & 0.40 & 1.00 & -0.57 & -0.37 & -0.52 & -0.49 \\
\hline
\end{tabular}

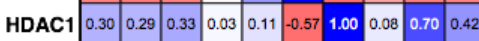

HDAC2 $-0.06-0.01-0.10-0.33-0.37-0.37 \quad 0.08$ 1.00 -0.11 - 0.05

\begin{tabular}{lllllllllll} 
HDAC3 & 0.48 & 0.46 & 0.53 & 0.16 & 0.08 & -0.52 & 0.70 & -0.11 & 1.00 & 0.19 \\
\hline
\end{tabular}

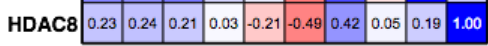
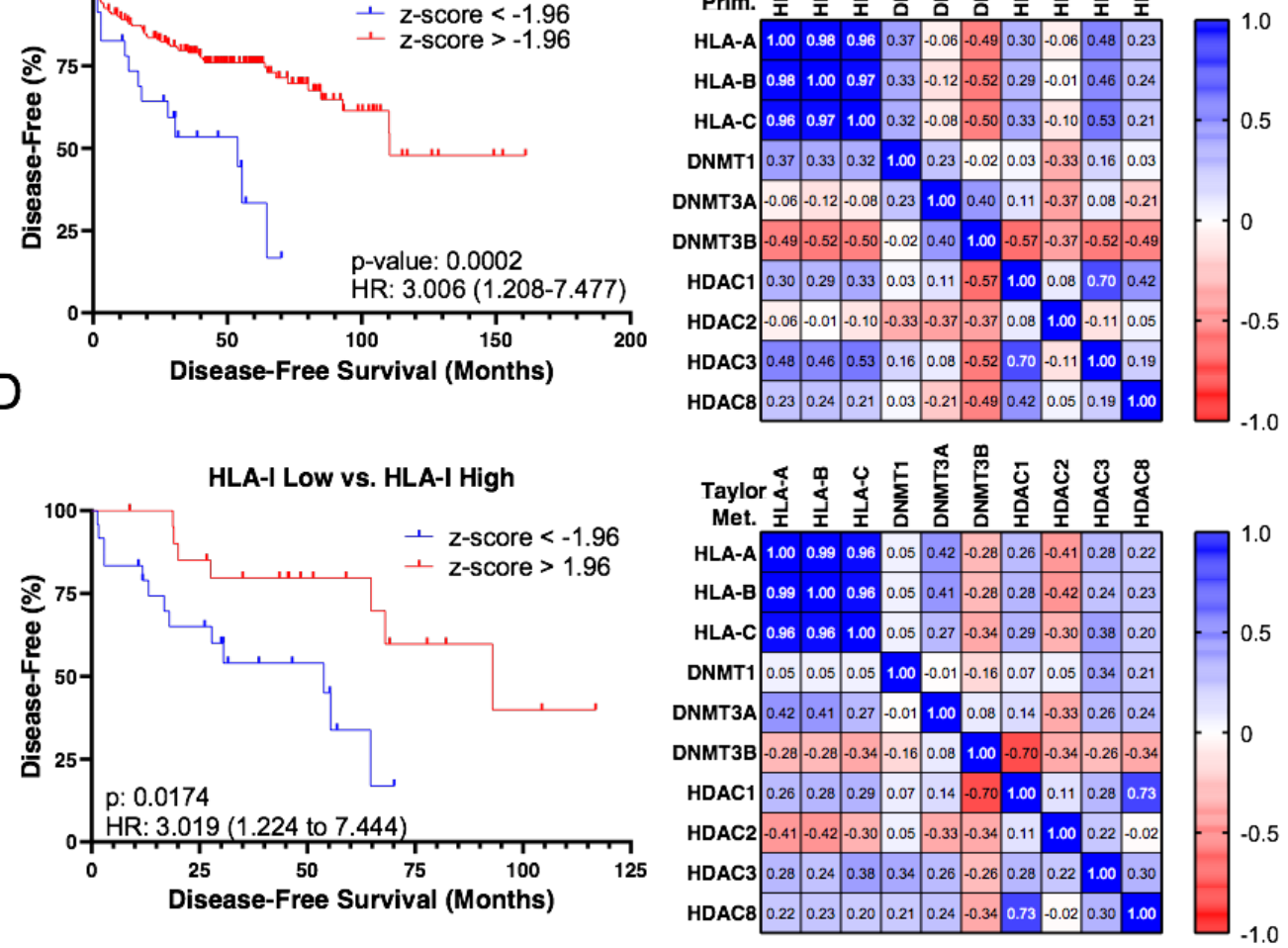

$E$

PRAD Primary

- Taylor Primary

- Taylor Metastatic
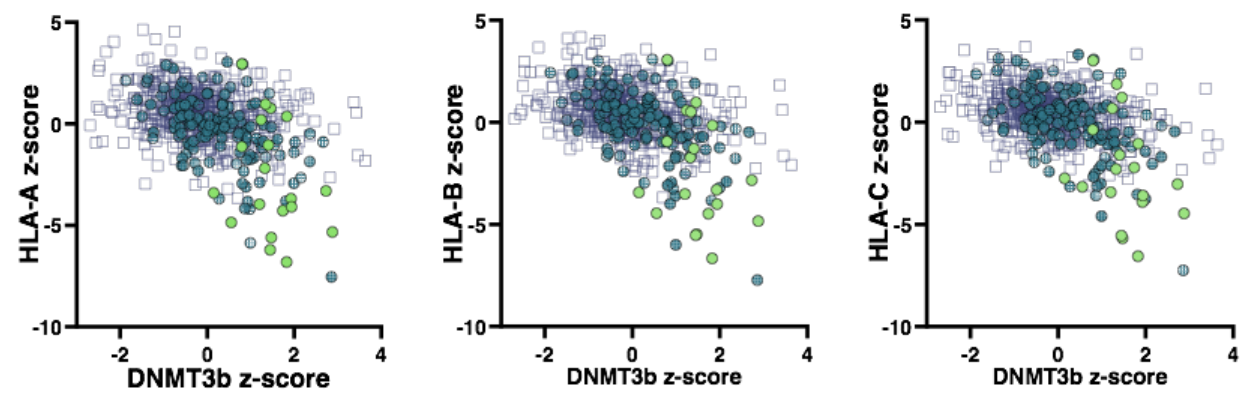
Figure 1. Genomic alterations and gene expression of HLA-I in primary and metastatic prostate adenocarcinoma. A) HLA-A, HLA-B, and HLA-C gene expression in the PRAD, and Taylor data set represented as z-scores relative to normal samples in the respective study. Dotted lines are at $+/-1.96$. Line and error bars represent mean and $95 \%$ confidence interval. B) Correlation matrix of gene expression of HLA-I genes and epigenetic modifying proteins in PRAD dataset. C) Kaplan-Meier curves showing disease-free survival time for patients in the Taylor data set with low expression (zscore $<-1.96$ ) vs. high expression (z-score > 1.96) of any HLA-I gene. D) Kaplan-Meier curves showing disease-free survival time for patients in the Taylor data set with low HLA-I expression in any HLA-I gene (z-score < -1.96) vs. all other patients (z-score > 1.96). E) Correlation of expression of DNMT3b to HLA-I genes. 
Figure 2
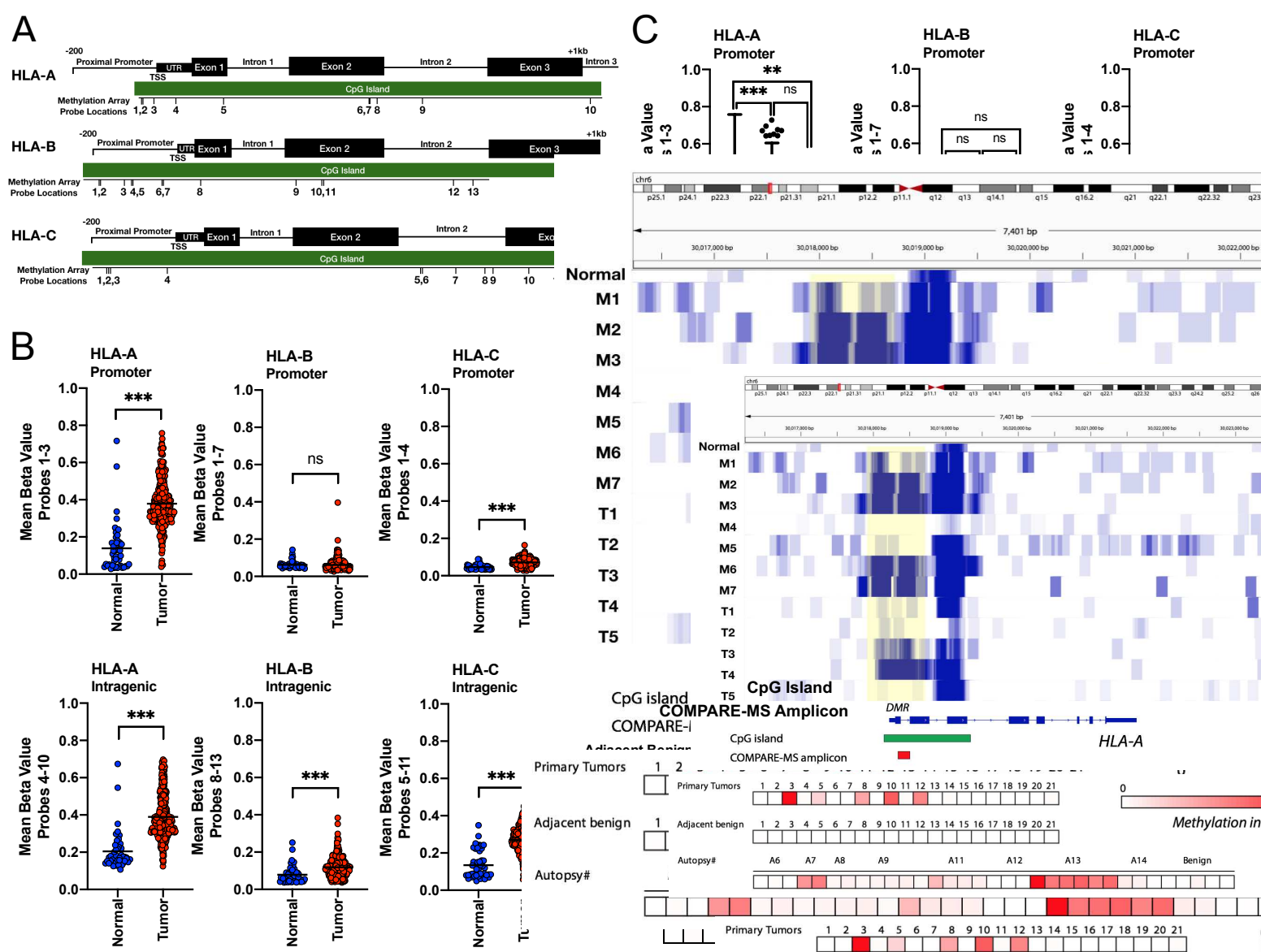

Cpg Island

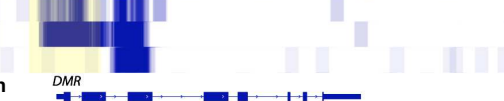

COMPARE-MS Amplicon

\section{Adjacent Benign}

Patient\# $\begin{array}{llllllllllllllllllll}2 & 3 & 4 & 5 & 6 & 7 & 8 & 9 & 10 & 11 & 12 & 13 & 14 & 15 & 16 & 17 & 18 & 19 & 20 & 21\end{array}$

Primary Tumors

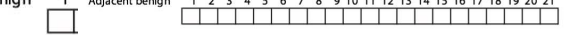

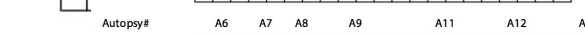

Methylation index

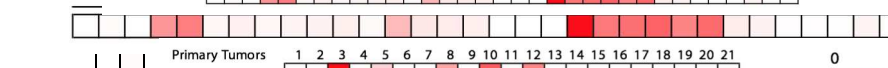

E
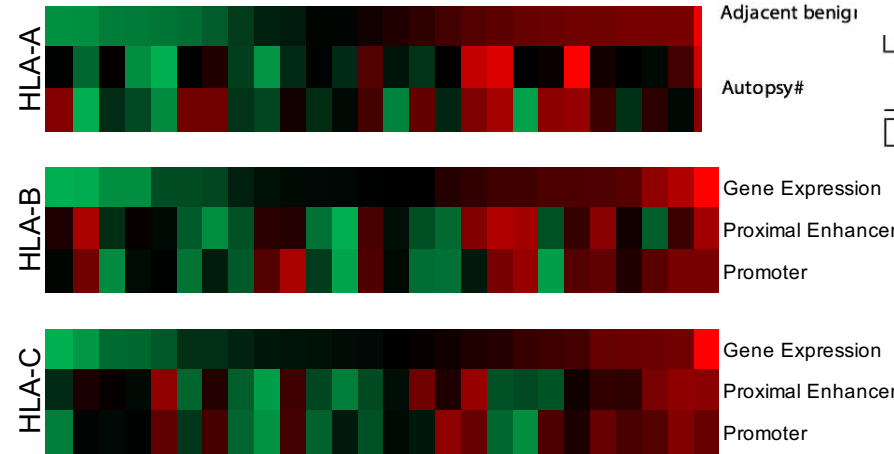

\section{F $\rightarrow$ Proximal Enhancer $\rightarrow$ Promoter}
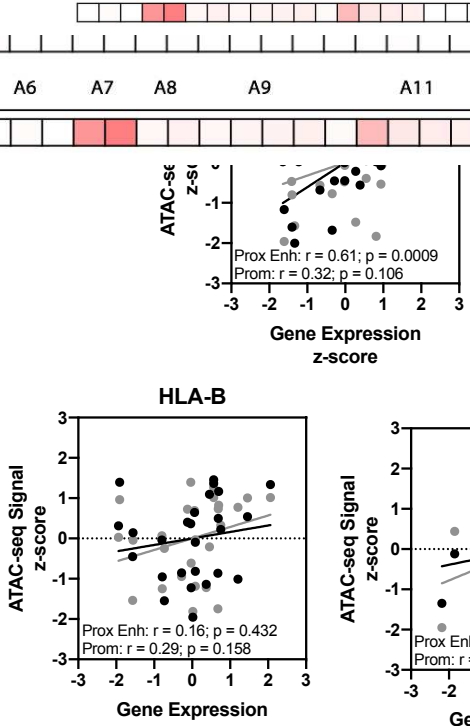

z-score

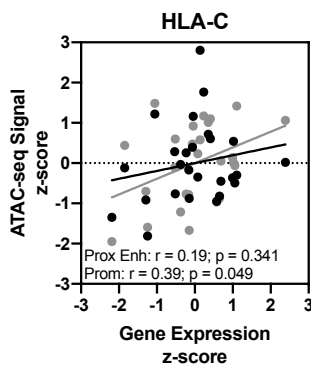


Figure 2. DNA methylation in HLA-I genes in prostate cancer. A) Approximate locations of probes within the HLA-I CpG islands. B) Methylation levels in the promoter or intragenic regions of the HLA-I CpG islands in normal and primary tumor samples represented by mean beta value determined by microarray analysis. C) Methylation levels in the promoter or intragenic regions of the HLA-I CpG islands in primary tumor samples stratified by gene expression level. Low expression: $z$-score $<-1.96$, med expression $1.96<z$-score $>-1.96$, high expression: z-score $>1.96$. D) Detection of HLA-A methylation in primary, adjacent benign, and metastatic lesions by COMPAREMS. Location of amplicon is indicated. E) ATAC-seq from PRAD data set at two genomic regions in each HLA-I gene. F) Correlation of ATAC-seq signal to gene expression in each HLA-I gene. $* p<0.05, * * p<0.01, * * * p<0.001$. 
Figure 3.

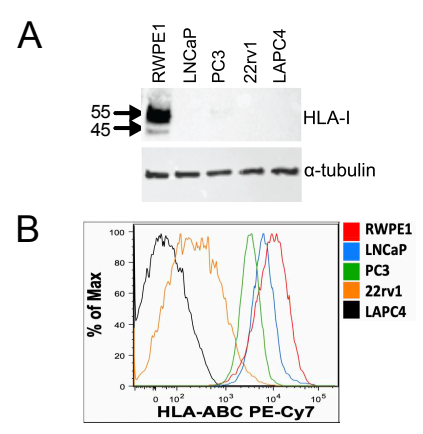

E

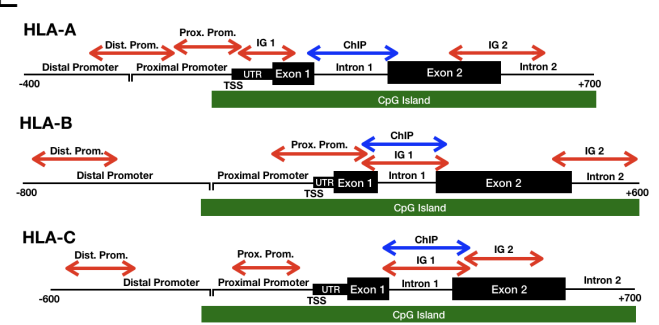

C

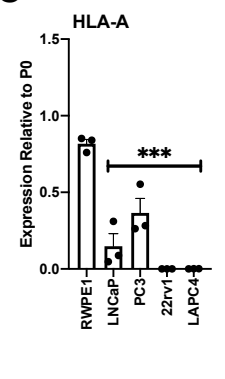

$G$

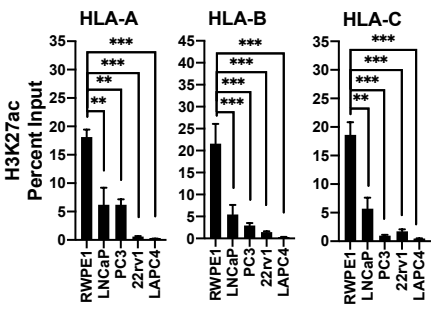

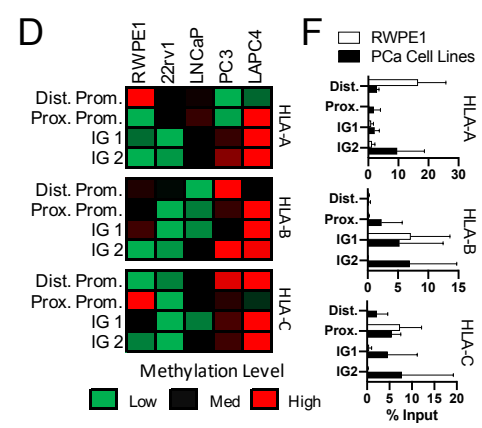

$\mathrm{H}$

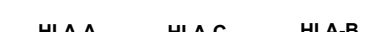

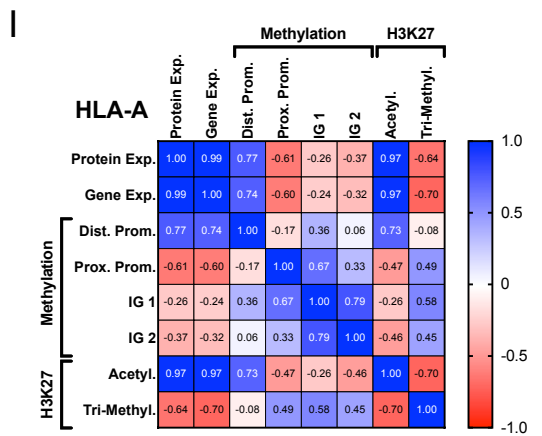
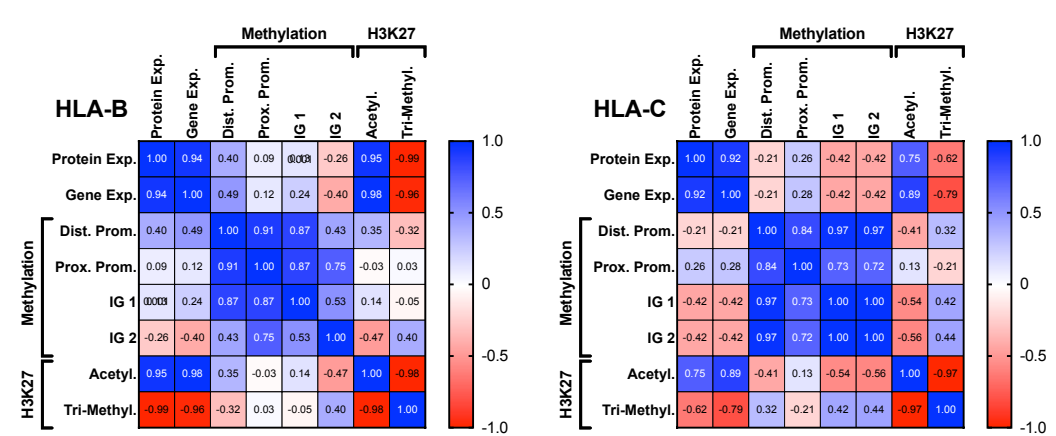

Figure 3. Repressive epigenetic signatures are associated with the HLA-I genes in cell lines with low HLA-I expression. HLA-I protein and gene expression was measured in 4 prostate cancer cell lines (LNCaP, 22rv1, PC3, and LAPC4) and a normal prostate epithelial cell line, RWPE1. A) Western blot analysis of HLA-I expression in whole cell lysates with alpha-tubulin as a loading control. B) Flow cytometry analysis of extracellular HLA-I expression. C) Gene expression of HLA-A, HLA-B, and HLA-C measured by qPCR relative to the housekeeping gene RPLPO (P0).

D) Heat map showing DNA methylation levels in LNCaP, 22rv1, PC3, LAPC4, and RWPE1 in four regions of the HLA-I. E) Primer locations for DNA methylation and histone modification analyses. F) Percent input of RWPE1 methylation and averaged methylation percent input in prostate cancer (PCa) cell lines. $(\mathrm{G}, \mathrm{H}) \mathrm{ChIP}$ with antibodies targeting H3K27ac $(\mathrm{G})$ and H3K27me3 $(\mathrm{H})$ in LNCaP, 22rv1, PC3, and LAPC4 compared to RWPE1. I) Correlation matrix of HLA-I gene and protein expression, DNA methylation levels, and histone modifications in LNCaP, 22rv1, PC3, LAPC4, and RWPE1. 
Figure 4.
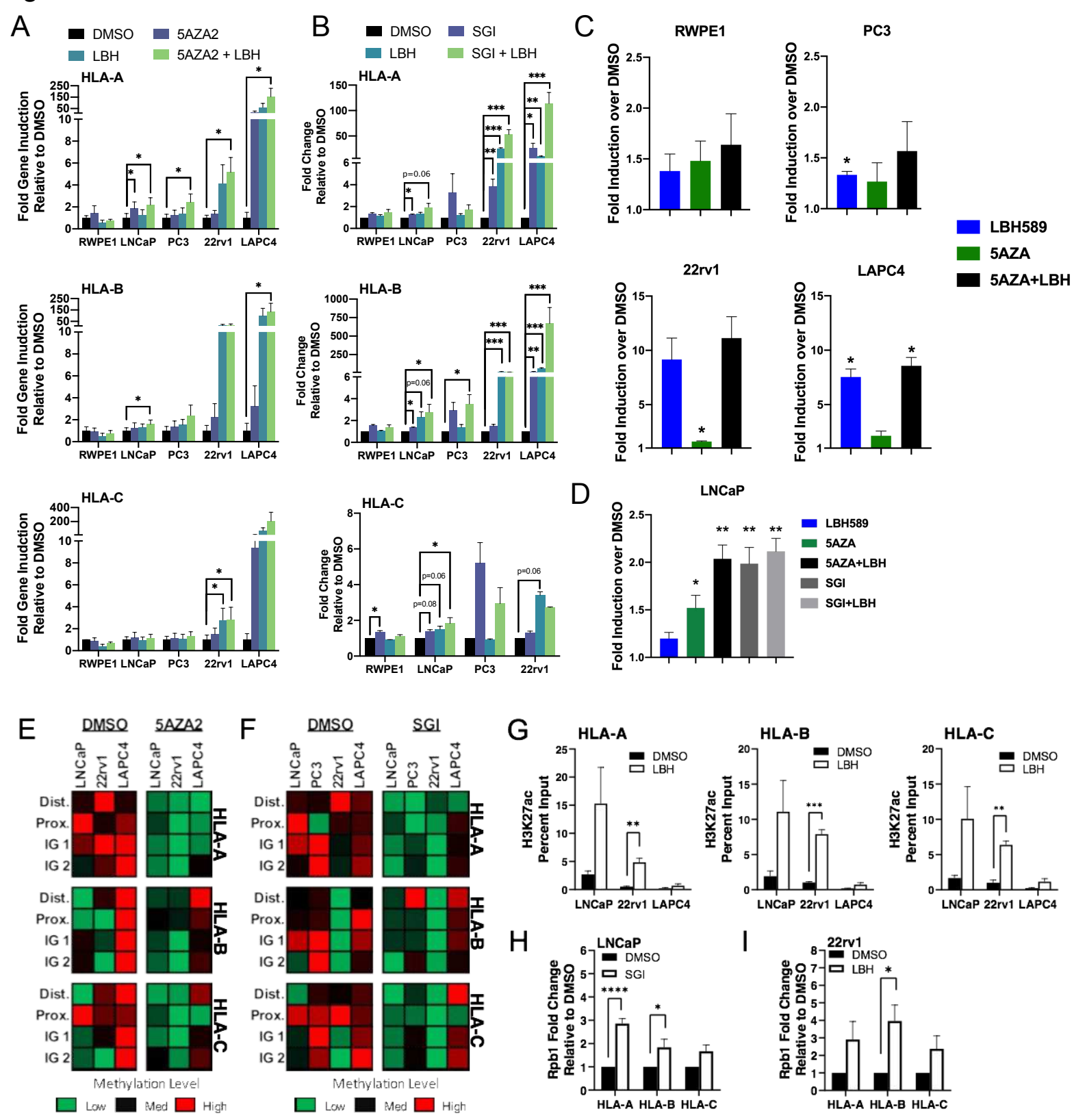

Figure 4. Pharmacological inhibition of DNMTs and HDACs induces HLA-I gene and protein expression. Gene expression changes in HLA-A, HLA-B, and HLA-C in response to A) $5 A Z A 2$ and $L B H$ or B) SGI and $L B H$ alone and in combination. Data is from 3 independent experiments and shown as fold change relative to DMSO treated cells. LAPC4 was excluded for HLA-C fold change analysis because HLA-C was undetectable in DMSO treated LAPC4 cells. Error bars represent SEM. C) Induction of HLA-I protein expression on the cell surface in indicated cell lines in response to 5AZA2 and LBH treatment alone and in combination. D) Induction of HLA-I protein expression on the cell surface of LNCaP cells in response to 5AZA2, SGI, or LBH alone and in 
combination. Flow cytometry data in (C) and (D) is expressed as fold-over induction of Median Fluorescent Intensity normalized to DMSO treated cells from at least three independent experiments. Mean +/- SEM. E) Heat map showing methylation levels in DMSO or 5AZA2 treated cells in four regions of the HLA-I genes from three independent experiments. F) Heat map showing methylation levels in DMSO or SGI treated cells in four regions of the HLA-I genes from two independent experiments. G) ChIP using antibodies to H3K27ac and H3K27me3 in LNCaP, 22rv1, and LACP4 cells treated with DMSO or LBH. H) ChIP using an antibody to RPB1 in LNCaP cells treated with DMSO or SGI. I) ChIP using an antibody to RPB1 in 22rv1 cells treated with DMSO or LBH. All ChIP data is representative of three independent experiments. For heat maps: Each heat map row was generated independent of other rows. Comparisons can be made between cell lines and treatment groups for each gene region individually, but not between different gene regions. $* p<0.05, * * p<0.01, * * * p<0.001$. 
Figure 5.

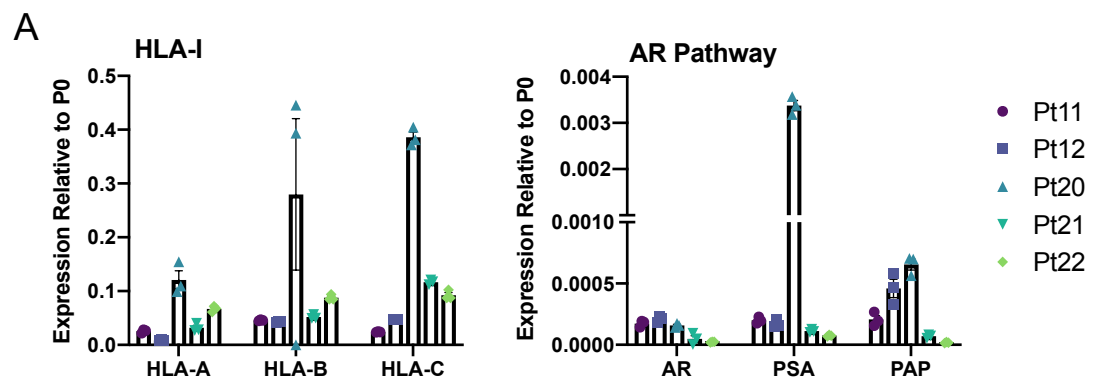

B
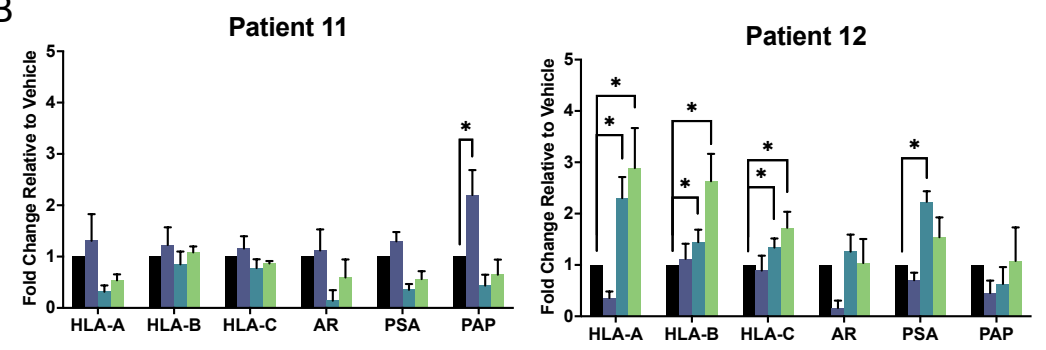

- DMSO

10بM 5AZA2

- 100nM LBH

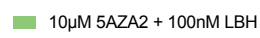
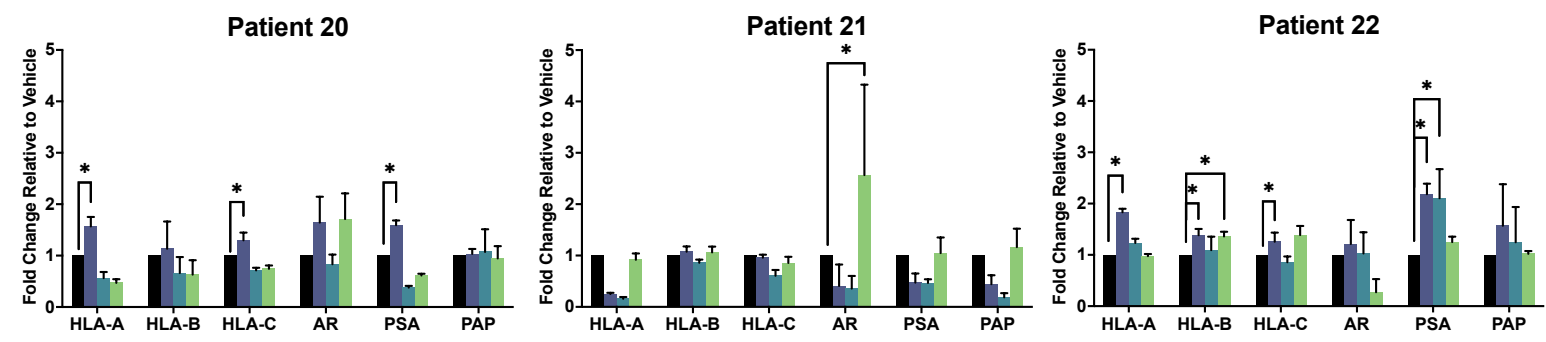

Figure 5. HLA-I expression and response to DNMT and HDAC inhibition ex vivo. A) Gene expression analysis of the HLA-I genes and prostate specific elements of the AR pathway in prostate tumor tissue cultured ex vivo. B) Gene expression analysis of induction of HLA-I and AR pathway elements in ex vivo tissue treated with DMSO, 5AZA2, LBH, or 5AZA2+LBH. Error bars represent SD. * $p<0.05$. 
Figure 6.

A

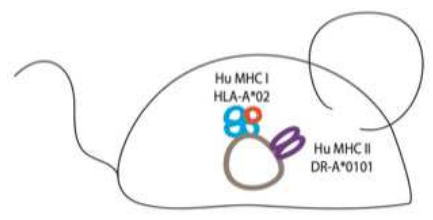

HHD/A02 mouse

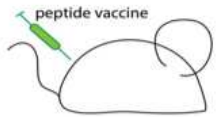

PSMA vaccination
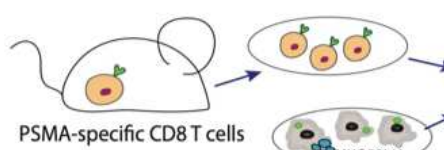

pre-treated $\mathrm{A}^{*} 02 \mathrm{PC}$ co-culture

B

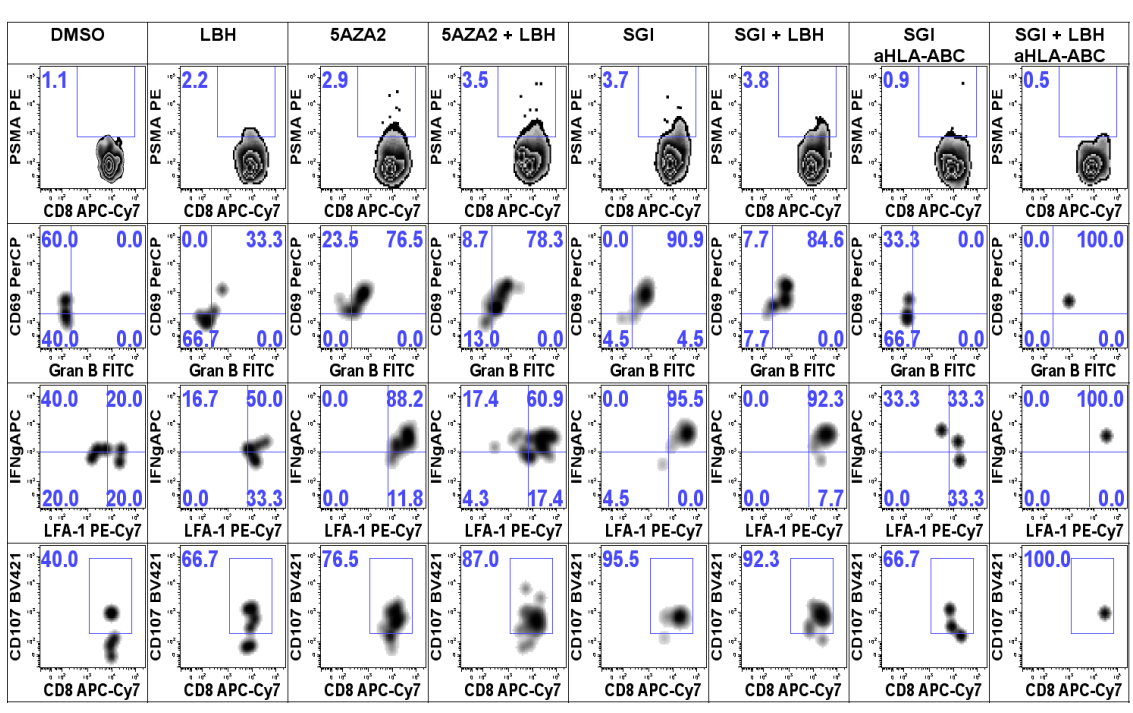

C

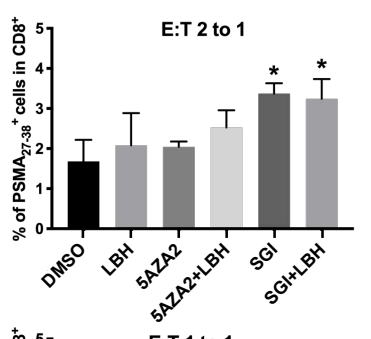

of tumor-specific

$\stackrel{T}{\mathrm{~T} \text { cell sentinels }}$

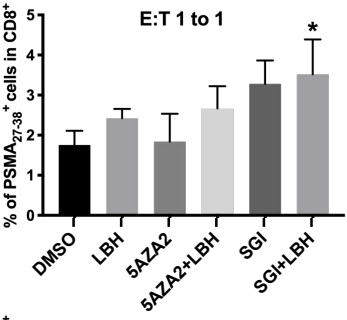

Un 57 UnVacc E:T 1to1

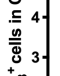
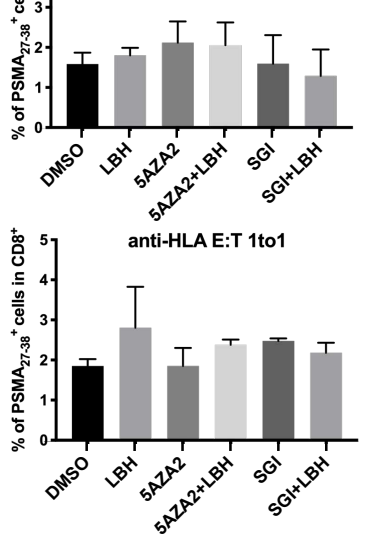

Figure 6. DNMT and HDAC inhibition in tumor cells increases co-cultured T-cell activation. A) Vaccination and co-culture scheme to analyze PSMA $27-38$-specific T-cell response to $\mathrm{LNCaP}$ cells treated with epigenetic modifying agents. B) Plots represent the rare-event cell analysis of PSMA $27-38$-specific T-cells to assess the cytolytic features. Top row shows $\mathrm{PSMA}_{27-38}$ tetramer binding on the total $\mathrm{CD} 8^{+}$splenocyte subset. $\mathrm{PSMA}_{27-38}$ tetramer ${ }^{+}$cells are projected in rows 2-4 and gate frequencies are expressed as a percentage of total PSMA $27-38 \mathrm{CD}^{+} \mathrm{T}$-cells. Plots represent expression of activation markers CD69, LFA-1, IFN $\gamma$, granzyme B, and CD107 (LAMP1). C) Graphs represent the frequency of $P S M A_{27-38}$ pentamer positive cells within the total $C D 8^{+} \mathrm{T}$ cell population after co-culture with $\mathrm{LNCaP}$ cells treated with epigenetic modifying agents. Splenocytes were co-cultured with $\mathrm{LNCaPs}$ in ratios of 2:1 and 1:1 PSMA $27-38$ tetramer ${ }^{+} \mathrm{CD}^{+}{ }^{+} \mathrm{T}$-cell Effector to tumor Target (E:T). In control conditions, splenocytes from unvaccinated mice were co-cultured with $\mathrm{LNCaPs}$ treated with epigenetic modifying agents or HLA-I as blocked by an anti-HLA-A,B,C blocking antibody. Error bars represent SD. * $p<0.05$. 
Figure 7.
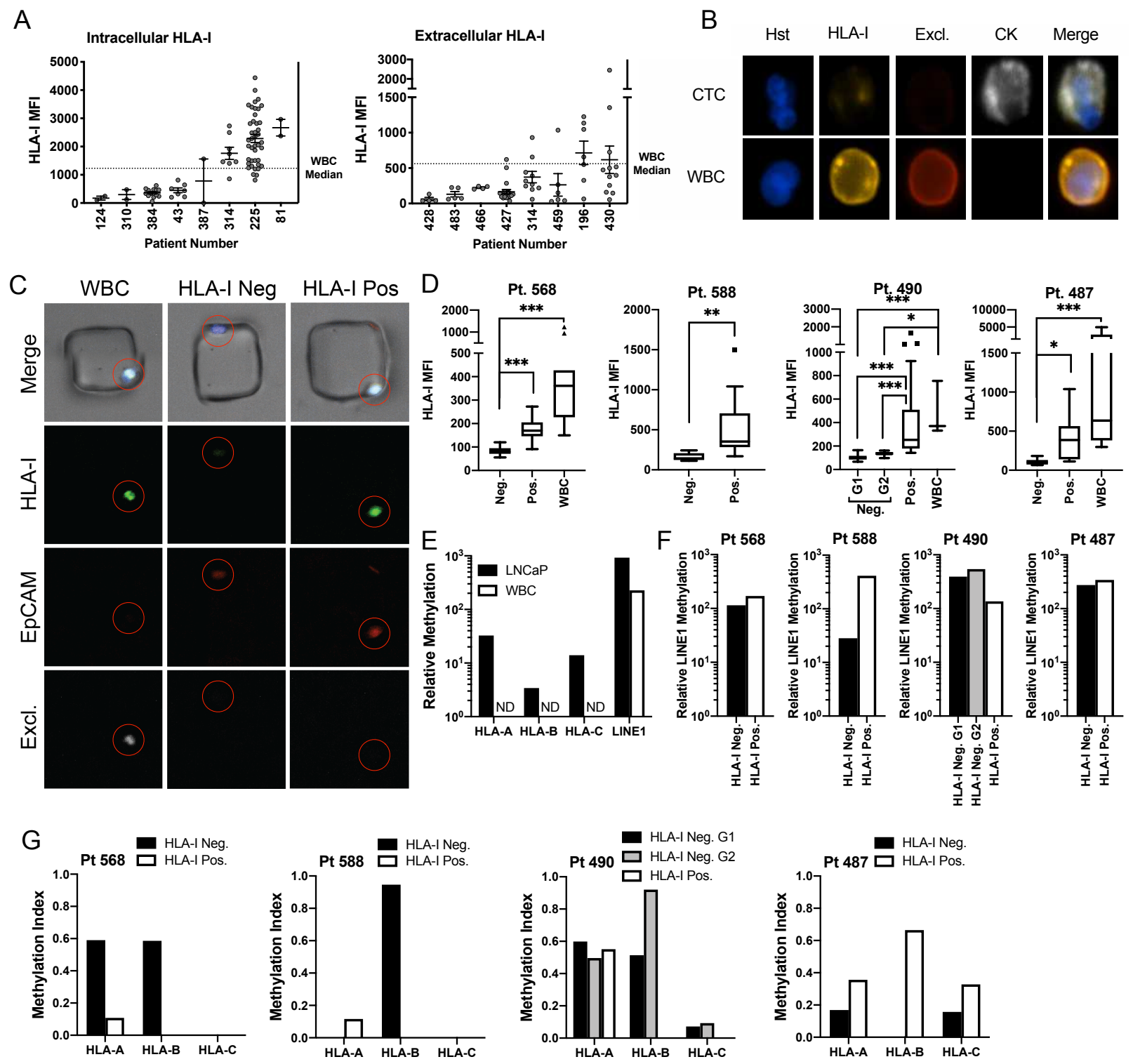

Figure 7. HLA-I expression and methylation in circulating tumor cells. A) Intra- and extracellular HLA-I protein expression in circulating tumor cells from two cohorts of 8 patients with prostate cancer. Dotted line represents median HLA-I expression in all patient matched WBCs. B) Representative 40x images of a CTC with low HLA-I expression and a WBC near the median expression from the total HLA-I group. Excl.: CD45/CD34/CD11b/CD27. C) Representative 10x images of HLA-I negative and HLA-I positive CTCs and a WBC in the single cell aspirator microwells (pt. 490). Circles indicate location of single cells. D) Mean fluorescent intensity (MFI) of HLA-I in each CTC population and matched WBCs (where available). Approximately 10-15 CTCs were chosen to be aspirated from each group. E) HLA-I methylation in 10 WBCs and 10 
LNCaP cells purified by single-cell aspiration. F) Relative LINE1 methylation in groups of approximately 10-15 CTCs purified by single-cell aspiration. G) HLA-I methylation in HLA-I positive and negative groups of approximately 10-15 CTCs purified by single cell aspiration represented with a Methylation Index relative to methylation in $\mathrm{LNCaP}$ cells. $* p<0.05, * * p<0.01, * * * p<0.001$. 


\section{REFERENCES}

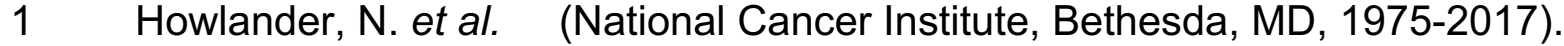

2 Kelly, S. P., Anderson, W. F., Rosenberg, P. S. \& Cook, M. B. Past, Current, and Future Incidence Rates and Burden of Metastatic Prostate Cancer in the United States. Eur Urol Focus 4, 121-127, doi:10.1016/j.euf.2017.10.014 (2018).

3 Boettcher, A. N. et al. Past, Current, and Future of Immunotherapies for Prostate Cancer. Front Oncol 9, 884, doi:10.3389/fonc.2019.00884 (2019).

4 Schweizer, M. T. \& Drake, C. G. Immunotherapy for prostate cancer: recent developments and future challenges. Cancer Metastasis Rev 33, 641-655, doi:10.1007/s10555-013-9479-8 (2014).

5 Hanahan, D. \& Weinberg, R. A. Hallmarks of cancer: the next generation. Cell 144, 646-674, doi:10.1016/j.cell.2011.02.013 (2011).

6 Seliger, B. Molecular mechanisms of HLA class I-mediated immune evasion of human tumors and their role in resistance to immunotherapies. HLA 88, 213-220, doi:10.1111/tan.12898 (2016).

7 Lee, J. H. et al. Transcriptional downregulation of MHC class I and melanoma de- differentiation in resistance to PD-1 inhibition. Nature communications 11, 1897-1897 (2020).

8 Fares, C. M., Van Allen, E. M., Drake, C. G., Allison, J. P. \& Hu-Lieskovan, S. Mechanisms of Resistance to Immune Checkpoint Blockade: Why Does Checkpoint Inhibitor Immunotherapy Not Work for All Patients? American Society of Clinical Oncology Educational Book, 147-164 (2019).

9 Garrido, F., Aptsiauri, N., Doorduijn, E. M., Garcia Lora, A. M. \& van Hall, T. The urgent need to recover MHC class I in cancers for effective immunotherapy. Curr Opin Immunol 39, 44-51, doi:10.1016/j.coi.2015.12.007 (2016).

10 Blum, J. S., Wearsch, P. A. \& Cresswell, P. Pathways of antigen processing. Annu Rev Immunol 31, 443-473, doi:10.1146/annurev-immunol-032712-095910 (2013).

11 Vinay, D. S. et al. Immune evasion in cancer: Mechanistic basis and therapeutic strategies. Semin Cancer Biol 35 Suppl, S185-198, doi:10.1016/j.semcancer.2015.03.004 (2015).

12 Hicklin, D. J., Marincola, F. M. \& Ferrone, S. HLA class I antigen downregulation in human cancers: T-cell immunotherapy revives an old story. Mol Med Today $\mathbf{5}$, 178-186 (1999).

13 Blades, R. A., Keating, P. J., McWilliam, L. J., George, N. J. \& Stern, P. L. Loss of HLA class I expression in prostate cancer: implications for immunotherapy. Urology 46, 681-687, doi:10.1016/s0090-4295(99)80301-x (1995).

14 Kitamura, $\mathrm{H}$. et al. Down-regulation of HLA class I antigens in prostate cancer tissues and up-regulation by histone deacetylase inhibition. $J$ Urol 178, 692-696, doi:10.1016/j.juro.2007.03.109 (2007).

15 Ruggero, K., Farran-Matas, S., Martinez-Tebar, A. \& Aytes, A. Epigenetic Regulation in Prostate Cancer Progression. Curr Mol Biol Rep 4, 101-115, doi:10.1007/s40610-018-0095-9 (2018).

16 Zhao, S. G. et al. The DNA methylation landscape of advanced prostate cancer. 52, 778-789 (2020). 
17 Gravina, G. L. et al. Increased levels of DNA methyltransferases are associated with the tumorigenic capacity of prostate cancer cells. Oncol Rep 29, 1189-1195, doi:10.3892/or.2012.2192 (2013).

$18 \mathrm{Kim}$, N. H., Kim, S. N. \& Kim, Y. K. Involvement of HDAC1 in E-cadherin expression in prostate cancer cells; its implication for cell motility and invasion. Biochem Biophys Res Commun 404, 915-921, doi:10.1016/j.bbrc.2010.12.081 (2011).

19 Ye, Q. et al. Hypermethylation of HLA class I gene is associated with HLA class I down-regulation in human gastric cancer. Tissue Antigens 75, 30-39, doi:10.1111/j.1399-0039.2009.01390.x (2010).

20 Nie, Y. et al. DNA hypermethylation is a mechanism for loss of expression of the HLA class I genes in human esophageal squamous cell carcinomas. Carcinogenesis 22, 1615-1623 (2001).

21 Liao, Y. \& Xu, K. Epigenetic regulation of prostate cancer: the theories and the clinical implications. Asian J Androl 21, 279-290, doi:10.4103/aja.aja_53_18 (2019).

22 Villanueva, L., Álvarez-Errico, D. \& Esteller, M. The Contribution of Epigenetics to Cancer Immunotherapy. Trends Immunol 41, 676-691, doi:10.1016/j.it.2020.06.002 (2020).

23 Heninger, E. et al. Inducible expression of cancer-testis antigens in human prostate cancer. Oncotarget 7, 84359-84374, doi:10.18632/oncotarget.12711 (2016).

24 Carretero, F. J. et al. Frequent HLA class I alterations in human prostate cancer: molecular mechanisms and clinical relevance. Cancer Immunol Immunother 65, 47-59, doi:10.1007/s00262-015-1774-5 (2016).

25 Taylor, B. S. et al. Integrative genomic profiling of human prostate cancer. Cancer Cell 18, 11-22, doi:10.1016/j.ccr.2010.05.026 (2010).

26 Mottahedeh, J. et al. CD38 is methylated in prostate cancer and regulates extracellular NAD+. Cancer \& metabolism 6, 13 (2018).

27 Yegnasubramanian, S., Lin, X., Haffner, M. C., DeMarzo, A. M. \& Nelson, W. G. Combination of methylated-DNA precipitation and methylation-sensitive restriction enzymes (COMPARE-MS) for the rapid, sensitive and quantitative detection of DNA methylation. Nucleic Acids Res 34, e19, doi:10.1093/nar/gnj022 (2006).

28 Yegnasubramanian, S. et al. DNA hypomethylation arises later in prostate cancer progression than $\mathrm{CpG}$ island hypermethylation and contributes to metastatic tumor heterogeneity. Cancer Res 68, 8954-8967, doi:10.1158/0008-5472.can-076088 (2008).

29 Wise, D. R. et al. Dickkopf-1 Can Lead to Immune Evasion in Metastatic Castration-Resistant Prostate Cancer. JCO Precision Oncology, 1167-1179 (2020).

30 Yegnasubramanian, S. et al. Chromosome-wide mapping of DNA methylation patterns in normal and malignant prostate cells reveals pervasive methylation of gene-associated and conserved intergenic sequences. BMC Genomics 12, 313, doi:10.1186/1471-2164-12-313 (2011). 
31 Chen, Z., Wang, L., Wang, Q. \& Li, W. Histone modifications and chromatin organization in prostate cancer. Epigenomics 2, 551-560, doi:10.2217/epi.10.31 (2010).

32 Seligson, D. B. et al. Global histone modification patterns predict risk of prostate cancer recurrence. 435, 1262-1266 (2005).

33 ENCODE Project Consortium. An integrated encyclopedia of DNA elements in the human genome. Nature 489, 57-74, doi:10.1038/nature11247 (2012).

34 Gkountela, S. et al. Circulating Tumor Cell Clustering Shapes DNA Methylation to Enable Metastasis Seeding. Cell 176, 98-112 e114, doi:10.1016/j.cell.2018.11.046 (2019).

35 Caceres, G., Puskas, J. A. \& Magliocco, A. M. Circulating Tumor Cells: A Window Into Tumor Development and Therapeutic Effectiveness. Cancer Control 22, 167-176 (2015).

36 Lang, J. M., Casavant, B. P. \& Beebe, D. J. Circulating tumor cells: getting more from less. Sci Transl Med 4, 141ps113, doi:10.1126/scitranslmed.3004261 (2012).

37 Tokar, J. J. et al. Pairing Microwell Arrays with an Affordable, Semiautomated Single-Cell Aspirator for the Interrogation of Circulating Tumor Cell Heterogeneity. SLAS Technol 25, 162-176, doi:10.1177/2472630319898146 (2020).

38 Sperger, J. M. et al. Integrated analysis of multiple biomarkers from circulating tumor cells enabled by exclusion-based analyte isolation. Clin Cancer Res 23, 746-756, doi:10.1158/1078-0432.ccr-16-1021 (2017).

39 Pezzi, H. M. et al. Versatile exclusion-based sample preparation platform for integrated rare cell isolation and analyte extraction. Lab Chip 18, 3446-3458, doi:10.1039/c8lc00620b (2018).

40 Rodems, T. S. et al. SEEMLIS: A Flexible Semi-Automated Method for Enrichment of Methylated DNA from Low Input Samples. In Review (2020).

41 Aryee, M. J. et al. DNA methylation alterations exhibit intraindividual stability and interindividual heterogeneity in prostate cancer metastases. Sci Trans/ Med $\mathbf{5}$, 169ra110, doi:10.1126/scitranslmed.3005211 (2013).

42 Yang, Y. A. \& Yu, J. EZH2, an epigenetic driver of prostate cancer. Protein Cell 4, 331-341, doi:10.1007/s13238-013-2093-2 (2013).

43 Yegnasubramanian, S. et al. Hypermethylation of $\mathrm{CpG}$ islands in primary and metastatic human prostate cancer. Cancer Res 64, 1975-1986 (2004).

44 Kortenhorst, M. S. et al. Analysis of the genomic response of human prostate cancer cells to histone deacetylase inhibitors. Epigenetics 8, 907-920, doi:10.4161/epi.25574 (2013).

45 Mao, W. et al. Immunogenicity of prostate cancer is augmented by BET bromodomain inhibition. J Immunother Cancer 7, 277, doi:10.1186/s40425-0190758-y (2019).

46 Cheng, Y. et al. Targeting epigenetic regulators for cancer therapy: mechanisms and advances in clinical trials. Signal Transduct Target Ther 4, 62, doi:10.1038/s41392-019-0095-0 (2019).

47 Rothbart, S. B. \& Baylin, S. B. Epigenetic Therapy for Epithelioid Sarcoma. Cell 181, 211, doi:10.1016/j.cell.2020.03.042 (2020). 
48 Italiano, A. Targeting epigenetics in sarcomas through EZH2 inhibition. $J$ Hematol Oncol 13, 33, doi:10.1186/s13045-020-00868-4 (2020).

49 Gao, J. et al. Integrative analysis of complex cancer genomics and clinical profiles using the cBioPortal. Sci Signal 6, pl1, doi:10.1126/scisignal.2004088 (2013).

50 Cerami, E. et al. The cBio cancer genomics portal: an open platform for exploring multidimensional cancer genomics data. Cancer Discov 2, 401-404, doi:10.1158/2159-8290.cd-12-0095 (2012).

51 Goldman, M. et al. The UCSC Xena platform for public and private cancer genomics data visualization and interpretation. BioRxiv, doi:10.1101/326470 (2019).

52 Corces, M. R. et al. The Chromatin Accessibility Landscape of Primary Human Cancers. Science (New York, N.Y.) 362, doi:10.1126/science.aav1898 (2018).

53 Diez-Villanueva, A., Mallona, I. \& Peinado, M. A. Wanderer, an interactive viewer to explore DNA methylation and gene expression data in human cancer. Epigenetics Chromatin 8, 22, doi:10.1186/s13072-015-0014-8 (2015).

54 Pascolo, S. et al. HLA-A2.1-restricted education and cytolytic activity of CD8(+) T lymphocytes from beta2 microglobulin (beta2m) HLA-A2.1 monochain transgenic $\mathrm{H}-2 \mathrm{Db}$ beta2m double knockout mice. J Exp Med 185, 2043-2051, doi:10.1084/jem.185.12.2043 (1997).

55 Casavant, B. P. et al. Paired diagnostic and pharmacodynamic analysis of rare non-small cell lung cancer cells enabled by the VerIFAST platform. Lab Chip 14, 99-105, doi:10.1039/c3lc50912e (2014).

56 Casavant, B. P. et al. The VerIFAST: an integrated method for cell isolation and extracellular/intracellular staining. Lab Chip 13, 391-396, doi:10.1039/c2lc41136a (2013).

57 Guckenberger, D. J. et al. Magnetic System for Automated Manipulation of Paramagnetic Particles. Anal Chem 88, 9902-9907, doi:10.1021/acs.analchem.6b02257 (2016). 


\section{Supplementary Files}

This is a list of supplementary files associated with this preprint. Click to download.

- 20210909HLASupplementaryFiguresNatComm.pdf

- TableS1GeneExpressionUpregulationandDownregulation.xlsx

- TableS2CorrelationHLAtoDNMTHDAC.xIsx

- TableS3CorrelationofMethylationandGeneExpression.xlsx

- TableS4MethylationArrayProbelnformation.xlsx

- TableS5Primers.xlsx

- TableS6Antibodies.xlsx

- TableS7SingleCellPatientCharacteristics.xIsx 